\title{
VERBO COME ELEMENTO DELLA FRASE IN FRIULANO ED IN FRANCESE*
}

\section{INTRODUZIONE}

Il presente lavoro è dedicato a una parte della sfera del verbo nel friulano letterario della seconda metà del ventesimo secolo, alla perifrasi verbale e alla locuzione verbale. Nella definizione della perifrasi verbale (PV) ho seguito il modello dei linguisti spagnoli Javier García Gonzáles, Fernández de Castro e Leonard Gómez Torrego, essendo il punto di partenza il mio postulato che la PV rappresenti in friulano una categoria grammaticale a parte o, almeno, una categoria in via di grammaticalizzazione. Parallelamente alla situazione in friulano osservo quella nel francese scritto contemporaneo, con lo scopo di constatare delle eventuali somiglianze e differenze. Tenendo in considerazione la realtà linguistica nella regione Friuli-Venezia Giulia, dove le interferenze fra l'italiano, il friulano ed il veneto sono tali che un non-friulanofono non riesce facilmente a distinguere quando si tratta di un sintagma di origine friulana e quando di un calco sintattico sull'italiano, sembra opportuno, in numerosi casi, confrontare il sintagma friulano e quello francese anche con la variante corrispondente in italiano letterario moderno; inoltre, nel capitolo in cui sono trattati i cosiddetti tempi bicomposti, vengono citati alcuni esempi nelle diverse varietà venete.

Il corpus è assai ampio, prevalendovi le opere in friulano:

- due romanzi di Carlo Sgorlon, Prime di sere (1971) e Il dolfin (1982)

- l'originale francese del Petit Prince (1943) di Antoine de Saint-Exupéry, la sua versione friulana (1992), e quella italiana (1999)

- la versione friulana, francese ed italiana del Vangelo secondo Luca

- tre opere teatrali - Buje (1971) di Lelo Cjanton e Alviero Negro, Strumîrs e Zambarlàns (1978) di Alviero Negro, Mari di Vigjùt (1978) di Guido Michelutti

- quattro numeri della rivista La patrie dal Friûl (Ot.1997, Nov.1998, Av.-Set.1998, Març 2000).

Mi hanno indirizzato alla scelta di questo argomento due motivi. In primo luogo, la lingua friulana rappresenta una grande ricchezza di perifrasi verbali e di locuzioni verbali, cioè vi si sente una forte tendenza al modo d'esprimersi pittoresco, all'uso

Questo studio si basa sulla tesi di master dell'autore discussa il 30.08.2002 all'Università di Ljubljana (relatore: prof. dr. Mitja Skubic; gli altri due membri di commissione: prof. dr. Tjaša Miklič, prof. dr. Vladimir Pogačnik).

Ringrazio Tanja Rogovič e Eros Bičič per aver rivisto il testo in italiano. 
delle forme perifrastiche, sia nella lingua parlata che nei testi scritti. Il secondo motivo è che finora l'argomento non è mai stato soggetto di una sistematica ricerca scientifica seguita da un'analisi approfondita.

\section{DEFINIZIONE DELLA PERIFRASI VERBALE}

Inizio col riassumere in una frase la definizione della PV dei soprascritti linguisti che trattano lo stesso argomento in spagnolo (vd. Torrego, pp. 5-12; Gonzáles, p. 19): secondo loro la PV è un sintagma verbale ormai grammaticalizzato fino a vari gradi (parzialmente o completamente) oppure un sintagma verbale che sta entrando nel processo di grammaticalizzazione. Siccome non c'è differenza globale fra i meccanismi su cui è basato il funzionamento del sistema verbale spagnolo e quello friulano, si può accettare la stessa definizione come punto di partenza per la ricerca sulle PV in friulano. In entrambe le lingue, nonché in italiano ed in francese, si tratta del sintagma costruito di due elementi verbali. Se per i due elementi costitutivi i linguisti spagnoli usano i termini el auxiliar ed el verbo principal o auxiliado, a me invece sembra più opportuno denominarli la costante (CO) e la variabile (VA). Avendo scelto due termini matematici ho attribuito ai due elementi lo stesso peso, a differenza dei termini classici l'ausiliare ed il verbo principale che impongono automaticamente un rapporto di gerarchìa, privilegiando il secondo elemento rispetto al primo. La CO appare sempre a sinistra, la VA a destra. Anzi, non posso prendere a prestito l'affermazione del Gonzáles che »il primo elemento verbale è un verbo alla forma personale« (vd. Gonzáles, p. 19), perché i numerosi esempi nella mia ricerca provano che la $\mathrm{CO}$ può essere sia un verbo alla forma personale che impersonale (infinito, gerundio o participio passato).

(1) Eco. Al è inutil di stâ a dâti òrdins che tant tu fâs di to cjâf l'istès. (Str., 15)

(2) Eliseo al veve fan. Al tirà fûr de valîs l'ultin toc di une pagnoche grande comprade

a Napoli la sere prime, e al si metè a mangjâ. (Pr.di s., 15)

Nel (1) la CO stâ si presenta alla forma impersonale, cioè all'infinito. Nel (2), invece, la $\mathrm{CO}$ metisi si presenta alla forma personale, alla terza persona singolare del passato remoto.

La VA, al contrario, è di regola un verbo alla forma impersonale (infinito, gerundio o participio passato). Rivolgendoci agli stessi esempi possiamo constatare che le VA dâ e mangjâ sono ambedue all'infinito. Gli esempi (3) e (4) dimostrano l'uso del gerundio nella VA tradusi e del participio passato nella VA leâ:

(3) E ne al pâr che cualchidun i vei dât un valôr aes mês contis e puisiis... tant l'è vêr che $a$ stan tradusint lis primis 60 contis in doi libris... in Furlàn e Talian e un dut in Talian. (P., Av.-Set.1999, 23)

(4) Un blestemadôr, sar Pieri, ch'al va leât 'e berline par che il popul, ch'al è bielzà culì pront e parecjât, al puedi vergognâlu [...] (Buje, 25).

$\mathrm{Nel}$ caso in cui la $\mathrm{CO}$ si presenta come una forma verbale personale, parlo della PV tipica, mentre nel caso opposto introduco il termine la PV atipica. La PV tipica 
((2),(3),(4)) è dunque l'unione di una forma verbale personale (»verbo ausiliare coniugato«) ed una forma verbale impersonale (»non coniugabile«, immutabile), la PV atipica ((1)) essendo l'unione di due forme verbali impersonali. Il (1) rappresenta l'unione in cui entrambi, la $\mathrm{CO}$ e la VA, si presentano all'infinito. Accanto a questo tipo di unione è possibile trovare nel corpus anche alcuni esempi delle $\mathrm{PV}$ atipiche in cui si combinano la $\mathrm{CO}$ al gerundio e la VA all'infinito:

(5) Intant un pôs di oms, puartant sun tun jet un paralitic, a cirivin di fâlu passâ e di pojâlu denant di lui. No rivant a puartâlu dentri parvie de fole, a montàrin sul cuviert e lu molàrin jù cul jet pai cops, juste tal mieč là ch'al jere Gjesù. (Luche $-5,19)$

Anzi, sono assai numerosi i casi dell'unione del participio passato e dell'infinito:

(6) Une sere, finît di cenâ, al lè-fûr, parvìe ch'al jere masse adore par lâ a durmî. (Pr.di s., 97)

L'unione dell'infinito e participio passato, a suo turno, appare spesso:

(7) "Il popul furlan al à dentri di sè il desideri di contâ di plui e di jessi rapresentật miôr - al dîs ancje il coordenadôr dal »Programma Friuli« - . (P., Nov.1998, 5)

Ciascuno dei due elementi verbali della PV, la $\mathrm{CO}$ e la VA, contribuisce la sua parte all'entità che costituiscono. La CO determina il valore sintattico del sintagma (persona, numero, temporalità, modalità, aspetto, voce, Aktionsart), mentre la VA funziona prevalentemente come portatore del contenuto semantico. Il collegamento fra la $\mathrm{CO}$ e la VA può essere diretto, senza alcun elemento accessorio, collegando i due elementi verbali ((3),(4),(7)), nonché indiretto, cioè per mezzo della preposizione $((1),(2),(5),(6))$. A volte l'elemento di collegamento è una locuzione avverbiale:

(8) Ma l'aspiet plui impuartant de question al jere che il concet ch'o vevi vût fintremai cumò di Jole al jere daûr a mudâsi dal dut. (Il Dolfin, 115)

La PV jessi daûr a+infinito consiste nella CO jessi, la VA all'infinito, in questo caso mudâsi, e la locuzione avverbiale daûr a. La variante corrispondente in francese presenta la medesima struttura: être en train de+infinito. La CO e la VA sono gli elementi principali della PV. Accanto alla preposizione o alla locuzione avverbiale (se esistono) che rappresentano elementi accessori, la PV può anche contenere un complemento oggetto o un avverbio:

(9) In chê sere al stentave a cjapâ siun. Al tornave simpri a pensâ che nol jere plui il zingar ch'al durmive tes ostanìis, tai toglâz o magari sot di un arbul, come che j jere capitât une volte, jù pe Basse. (Pr.di s., 48)

(10) Mi semeave ch'al vès l'ande di un pastôr ch'al à piardudis lis sôs pioris e che al è daûr a cirîlis in eterno. (Il dolfin, 74)

$\mathrm{Nel}$ (9) l'elemento accessorio è l'avverbio simpri, mentre nel (10) il complemento oggetto, il pronome personale nella forma femminile plurale lis.

\section{DISTINZIONE FRA LA PERIFRASI VERBALE E LA LOCUZIONE VERBALE}

In certi casi è difficile distinguere fra la PV e la locuzione verbale perché i due tipi di sintagmi contengono due elementi verbali. In base alla mia analisi vengo alla 
conclusione che l'unico criterio adeguato è la prevalenza della dimensione sintattica su quella semantica, cioè nei casi in cui il valore sintattico del sintagma si dimostra più forte del suo carattere semantico, parliamo di una PV, mentre la predominanza del carattere semantico è la prova che si tratti di una locuzione verbale.

Inoltre, nel caso della PV il soggetto della CO e della VA è lo stesso, il sintagma quindi rappresenta un predicato, come si può vedere in tutti gli esempi da (1) a (10). C'è comunque un'eccezione a questa regola, il sintagma fâ +infinito, in cui il soggetto della CO non può essere che diverso di quello della VA:

(11) Al ridé, al tocjà la cuarde, al fasé girâ la cìdule. (Ex., 82)

"Il rit, toucha la corde, fit jouer la poulie.«, dunque la versione originale di Saint-Exupéry, conferma che il soggetto della CO fâ è »lui«, il soggetto della VA girâ »la cidule«. Anche dalla versione italiana della stessa frase, "Rise, toccò la corda, mise in moto la carrucola.« è evidente la presenza di due soggetti.

E'invece differente la situazione nel caso della locuzione verbale, dove il soggetto dei due elementi verbali può essere lo stesso oppure diverso. Occorre distinguere fra i sintagmi come olsâ a +infinito, tentâ di+infinito, bramâ di+infinito, etc., che secondo la struttura assomigliano alle $\mathrm{PV}$, riflettendo però chiaramente la prevalenza semantica su quella sintattica, ed i sintagmi come judâ cualchidun a+infinito, menâ cualchidun $a+$ infinito, permeti a cualchidun di+infinito etc. Nel presente lavoro uso il termine le locuzioni verbali semplici per il primo gruppo, dove si tratta di un solo soggetto, e le locuzioni verbali composte per il secondo, la cui caratteristica è la presenza di due soggetti. I sintagmi appartenenti al secondo gruppo si distinguono quindi dalle PV anche dal punto di vista della struttura, non solo per la dimensione prevalente della componente semantica. Per entrambi i gruppi introduco il termine le locuzioni verbali di tipo aperto, perché il secondo elemento verbale può essere qualunque lessema verbale, al fine di confrontarli con i sintagmi del tipo jessi a stâ, lassâ stâ, vê ce dî cun cualchidun, al vûl dî̀, etc., in cui né il lessema nel primo né quello nel secondo elemento verbale è sostituibile da un altro lessema. Possiamo denominarli le locuzioni verbali di tipo chiuso perché ciascuno di loro rappresenta una entità semanticamente fissa.

Può darsi che il medesimo sintagma funzioni in un determinato contesto come la PV, mentre in un altro si comporti come la locuzione verbale di tipo aperto. Ad esempio, visto che il verbo rivâ, nel (12), mantiene il suo valore semantico "giungere, muoversi fino ad un certo punto«, possiamo constatare che il sintagma rivâ a+infinito ha in questo contesto il puro valore di locuzione verbale:

(12) Disèimi, invezzit: sêso rivât a timp a fâj l'imbassade al cjastalt di Cjarisà? (Str., 13)

A differenza del (5), in cui rivâ ha un nuovo valore semantico, il sema »movimento« si vede già sostituito dal sema »riuscita«, abbiamo dunque da fare con la PV tipi$\mathrm{ca}$ al valore modale, esprimendo il conseguimento dello scopo. Non si tratta più del primo e secondo elemento verbale, ma della $\mathrm{CO}$ e la VA.

Propongo un confronto simile fra due esempi con il sintagma torna a+infinito: 
(13) L'astrònim al tornà a fâ la sô dimostrassion dal 1920 vistît dut elegant. (Ex.,21)

(14) Eliseo al fasè doi pàs sù e jù pe strade, par parâsi dal frêt, po al tornà a sentâsi sul cjaruz, taponant lis gjambis miôr ch'al podeve cu la manteline. (Pr.di s., 55)

Nel (13) torna ha preso il valore semantico iterativo, di conseguenza il sintagma come entità subisce automaticamente il carattere sintattico, la sua dimensione semantica è spinta in secondo piano. E' quindi evidente che si tratta della PV, in cui tornâ ha la funzione di CO. Nell'originale francese, in questo luogo appare il prefisso -re:

L'astronome refit sa démonstration en 1920, dans un habit très élégant.

Anche l'italiano utilizza, nell'espressione dell'aspetto iterativo, il medesimo prefisso, $-r i$ :

L'astronomo rifece la sua dimostrazione nel 1920 , con un abito molto elegante.

$\mathrm{Nel}$ (14), al contrario, tornâ rimane il portatore del suo valore semantico originale, cioè "andare verso il luogo da cui si è partito«, abbiamo dunque da fare con la locuzione verbale di tipo aperto.

\section{CLASSIFICAZIONE DELLE PERIFRASI VERBALI}

Per quanto riguarda la struttura, le PV friulane si possono dividere, secondo il

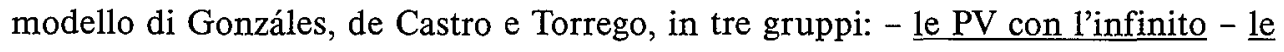

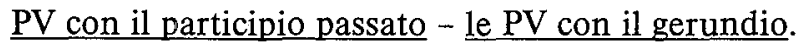

Il friulano dispone, come l'italiano ed il francese, di due PV con il gerundio: stâ +gerundio e lâ +gerundio. Entrambi servono ad attualizzare l'azione, a darle l'aspetto progressivo.

Possono essere considerati come PV con il participio passato:

1. i sintagmi verbali ormai completamente grammaticalizzati per cui nelle grammatiche classiche viene usato il termine $\underline{i}$ tempi composti. Si tratta dei sintagmi $v \hat{e}+$ part.pass. e jessi +part.pass. Riguardo alla struttura nonché al contenuto semantico dei sintagmi in questione (vê e.jessi non avendo più il loro valore semantico originale - caratteristica della $\mathrm{CO}$ ), essi si possono classificare fra le PV.

2. i sintagmi verbali, anche essi grammaticalizzati fino ad un alto livello, tradizionalmente caratterizzati come i mezzi per l'espressione della voce passiva: jessi +part.pass. e vigni + part.pass. Non sarebbe corretto affermare che jessi in funzione di copula abbia perso il sema "stato«, da questo punto di vista non abbiamo da fare con la vera $\mathrm{CO}$, però tenendo in considerazione il fatto che si tratta del mezzo che in numerosi casi dell'espressione del valore passivo non è sostituibile da nessun altro mezzo linguistico ed il fatto che sia estremamente esteso nell'uso orale e quello scritto, dobbiamo caratterizzarlo come una PV.

3. il sintagma lâ +part.pass. con il valore di obbligatorietà.

Le più numerose sono, come del resto nelle altre lingue romanze, le PV con l'infini-

to. Esse sono portatrici di valori semantici molto diversi, ad esempio quello di:

- aspetto (re)iterativo (tornâ a+infinito)

- conseguimento dello scopo (rivâ a+infinito) 
- fase dell'azione (stâ par+infinito, (s)comenzâ a+infinito, tacâ a+infinito, metisi a+infinito, continuâ a+infinito, finî di+infinito, fermâ di+infinito)

- attualizzazione dell'azione (stâ a+infinito, jessi daûr a +infinito)

- fattualità (fâ +infinito)

- obbligatorietà (vê di+infinito, scugnî +infinito) etc.

Secondo il livello di grammaticalizzazione che hanno ottenuto, possiamo dividere le PV friulane in tre gradi:

1. le PV completamente grammaticalizzate: vê +part.pass., jessi +part.pass., vignî +part.pass., lâ + part.pass., no stâ (a) +infinito (per l'espressione dell'imperativo negativo)

2. le PV parzialmente grammaticalizzate: stâ +gerundio, lâ +gerundio, jessi daûr a+infinito, fâ + infinito, stâ par + infinito, stâ a+infinito, rivâ a+infinito, tornâ a + infinito, vê di + infinito. La caratteristica comune delle $\mathrm{PV}$ in questione è che la $\mathrm{CO}$ mantiene in parte il suo valore semantico originale, l'uso del sintagma si è comunque amplificato a tal punto che il processo di grammaticalizzazione è ovvio: il carattere sintattico del sintagma sta crescendo, soppiantando progressivamente la sua dimensione semantica. Anzi, il parlante dispone sempre di un altro mezzo linguistico per l'espressione della stessa sfumatura di significato. Ad esempio, invece di tornâ a+infinito, può usare il verbo di pieno significato ed aggiungere l'avverbio »di gnûf«, oppure »ancjemò une volte«. Per esprimere il valore di vê di+infinito il parlante ha a disposizione il suo equivalente scugnî + infinito, a volte anche le locuzioni verbali $i$ vûl + part.pass., $i$ vûl +infinito, bisugne +infinito, covente +infinito, covente +part.pass.

3. i sintagmi verbali entrando nel processo di grammaticalizzazione $(=\mathrm{i}$ casi limitrofi):

a) quelli in cui la $\mathrm{CO}$ è un verbo indicando la fase dell'azione (stâ partinfinito, (s)comenzâ a+infinito, tacâ $a+$ infinito, metisi $a+$ infinito, continuâ $a+i n f i n i t o$, fermâ di $+i n f i n i-$ to, finî di+infinito, finî par+infinito)

b) usâ a+infinito per l'espressione dell'aspetto frequentativo (=un'azione abituale)

c) quelli con le $\mathrm{CO}$ podê, dovê, volê, scugnî, savê, per cui la grammatica tradizionale utilizza il termine $\underline{i}$ verbi modali o servili.

In 3. si tratta di casi in cui non è soddisfatta una condizione molto importante per l'esistenza della $P V$, cioè il valore semantico originale del verbo nella $C O$ non ha mutato, e da questo punto di vista sarebbe contraddittorio parlare delle PV. Anzi, l'orientamento semantico di tali sintagmi condiziona la loro diffusione universale, un segno chiaro del primo stadio del processo di grammaticalizzazione.

\section{GIUNTURE PERIFRASTICHE (GP)}

Torrego parla di cosiddette »agrupaciones perifrásticas« (vd. Torrego, p. 29), il fenomeno di giuntura di due o parecchie PV: la VA della prima ha allo stesso tempo la funzione della CO della seconda PV, la VA della quale può a suo turno prendere il ruolo della CO di una terza PV etc. Dal punto di vista sintattico la giuntura intera rappresenta un solo predicato. Ad esempio, il 
(15) Eliseo al viodeve ben ch'e jere dome une zerimonie, e cualchidun cjalant al podeve ancje mètisi a riduzzâ; ma lui la sintive tal profont [...] (Pr.di s., 98)

contiene la giuntura di due PV, podê+infinito esprimendo la possibilità e metisi a+infinito indicando la fase imminenziale dell'azione. La prima PV è tipica (la CO alla terza persona singolare dell'imperfetto), la seconda atipica (la $\mathrm{CO}$, che è allo stesso tempo la VA della PV tipica, all'infinito). In ogni giuntura perifrastica solamente la prima PV può essere tipica, cioè essa può incominciare con il verbo nella forma personale, la seconda e tutte le altre (se esistono) sono sempre atipiche perché non possono incominciare che con un verbo nella forma impersonale. Per l'elemento verbale nella forma impersonale che congiunge due PV, come mètisi nel (15), introduco il termine la costante statica (CS). Il termine sottolinea l'invariabilità, il carattere statico dell'elemento verbale posto fra la prima $\mathrm{CO}$ e la seconda VA. Possiamo constatare che si tratta in effetti solo di tre elementi costitutivi, cioè della $\mathrm{CO}$, della $\mathrm{CS}$ e della VA: $\mathrm{GP}=\mathrm{CO}+\mathrm{CS}+\mathrm{VA}$.

Ugualmente, jessi è la CS della GP nel

(16) »Meteisi ben tal cjâf chestis peraulis: il Fî dal om al sta par jessi consegnât tes mans dai oms «. (Luche - 9,44).

La CO è stâ all'indicativo presente e la VA consegnâ al participio passato.

5.1. Siccome nel presente lavoro ho classificato i sintagmi ormai completamente grammaticalizzati e tradizionalmente definiti con il termine tempi composti fra le PV e poiché il loro comportamento mostra incontestabilmente l'appartenenza a questa categoria grammaticale, posso venire alla conclusione che nel seguente esempio appaiono tre PV congiunte, con due CS, podût e continuâ:

(17) [...], pal fat ch'al jere daûr a intimpâsi e che nol varès podût continuâ a cjaminâ diluncvie lis stradis par simpri. (Il dolfin, 86).

All'interno di questa giuntura si presentano dunque due elementi statici, inflessibili, circondati dalla $\mathrm{CO}$ alla forma negativa del condizionale nol varès e la VA cjaminâ all'infinito.

5.2. Per analogia, è opportuno definire come giunture perifrastiche anche i sintagmi verbali per l'espressione della voce passiva quando essi appaiono ai tempi composti:

(18) La poesie e jere intitulade »Il gno paîs«, e e jere stade publicade sul »Strolic Furlan« pal an 1947 de Societât filologiiche, cu la liriche »L'agnul dal cis'cièl«. (P., Av.Set.1999, 17)

La PV tipica e jere stade si presenta come unione della $\mathrm{CO}$ jessi alla terza persona singolare dell'imperfetto e la VA jessi al participio passato, la PV atipica stade publicade essendo l'unione del medesimo participio passato (la VA della prima PV e la CO della seconda) e del participio passato publicade. Per mezzo dei simboli, la situazione nel (18) potrebbe riassumersi nel modo seguente:

$\mathrm{GP}=\mathrm{CO}+\mathrm{CS}+\mathrm{VA} ; \mathrm{CO}=$ jere, $\mathrm{CS}=$ stade, $\mathrm{VA}=$ publicade . 
Accorgendoci però che nel caso presente l'elemento di giuntura fra la $\mathrm{CO}$ e la VA prende la finale femminile singolare -de, non possiamo concludere che esso rappresenti un elemento assolutamente statico, sarebbe più opportuno definirlo come una costante semi-statica. Lo stesso vale per i casi in cui l'elemento di giuntura appare al plurale (maschile o femminile):

(19) Une des bausiis plui grandis inventadis par fâ gloti lis peresons ai cjargnêi e je stade chê di prometi che lis vuardiis e il personâl ausiliari aministratîf des gnovis peresons $a$ saressin stâts cjapâts sù mediant concors regjonâl, cul dirit di precedence justeapont pai residents tai cumuns de mont. (P., Ot.1997, 9)

5.3. Non sarebbe nemmeno possibile parlare di una sola PV ma della giuntura perifrastica quando lâ all'interno della PV lâ +part.pass. viene usato in un tempo composto, come nel (20) e nel (21):

(20) Da Cormòns al Nadisòn al è lât brusât squasi dut [...] e chel che nol à ruinât il fûc, lu à ruinât il taramòt [...] e la peste, po, 'e à fat ancjemò di piês [...] (Str., 71)

(21) I regjistris dai muarts a comencin dal 1650 cul volum cuart, chest al fâs pensâ ch' $a$ sedin lâts pierdûts i prins trê volums. (P., Av.-Set.1999, 18)

In entrambi gli esempi la PV lâ +part.pass. serve ad esprimere la deduzione logica, cioè la probabilità che un' azione si sia svolta nel passato.

5.4. I sintagmi verbali conosciuti sotto il nome di tempi bicomposti o sovracomposti (vd. Marchetti, p. 232) o le forme bicomposte (vd. C. Marcato, p. 48) in friulano rappresentano un tipo peculiare di giunture perifrastiche, perciò meritano un'attenzione particolare. In quindici sui diciassette esempi di tali sintagmi trovati nel corpus l'elemento di giuntura è la CO statica vût, in due casi si tratta invece della CO semistatica, la forma femminile singolare del medesimo participio passato, vude. La maggior parte degli esempi sono stati trovati nello Sgorlon, il resto nella rivista, mentre le opere drammatiche, il Piccolo Principe ed il Vangelo secondo Luca non contengono nessun esempio. In tali giunture perifrastiche la $\mathrm{CO}$ è sempre il verbo vê alla forma personale ed il lessema nella VA non può essere che un verbo transitivo oppure jessi:

(22) A' jerin duc' insieme te stànzie plui frescje de cjase, ros in muse. Eliseo ju veve sintûz a vosâ, e al pensave ch' a vessin vût fevelât di interès. Apene jentrât lui, a' vevin tasût a colp. (Pr.di s., 127-128)

(23) Ta chel timp istès, parâtri, mi pareve che la mê sostance di canai 'e partignis a chês costruzions lontanis squasit plui che no 'e cjase mê, come se in timps dismenteâz $\mathrm{j}$ vès abitât, e podopo 'o vès vût scugnût bandonâlis parvie di qualchi aveniment disgraciât. Postaj che chê impression si svilupàs dal fat che lenti-jù al veve vût stât il Tenent valdês. (Il dolfin, 59)

(24) Al procès a' vevin fevelât đi rabie bestiâl e di premeditazion. In paîs nissun nol podeve viòdilu parceche al jere rabiôs e prepotent, e duc' la vevin simpri vude piardude cun lui. Al veve robadis lis fantatis e lis feminis di chei altris, e duc' par pore a' vevin scugnût gloti e tasê par agn e agn; [...] (Pr.di s., 124) 
In due casi nel corpus le giunture perifrastiche in questione appaiono alla forma interrogativa, benché in realtà si tratti di domande retoriche:

(25) L'Omp cul orloi d' àur parcè mai varessial vût frequentât la mê cjase, lui ch' al vignive cuissà d' indulà, se no par chest? No savevi inmaginâmi nissune âtre reson. (Il dolfin, 114)

(26) Parcé varessie vût puartât zujatui di grant presit propit a mi, ch' in' vevi bielzà une stanze plene, e a lui nome robis di nuje tant par saurî la bocje? (Il dolfin, 53)

Non è stato trovato, al contrario, nessun esempio di tali giunture perifrastiche alla forma negativa.

\section{IMPIEGO DEI TEMPI BICOMPOSTI IN FRIULANO}

L'argomento rappresenta uno dei fenomeni meno chiariti nella grammatica friulana. Si tratta del fenomeno bene conosciuto alla sfera linguistica galloromanza (francese, francoprovenzale, occitanico), alla parte marginale di quella iberoromanza (catalano), nonché a certe varietà di ladino dolomitico (le parlate di Livinallongo, Rocca Pietore), al soprasilvano e, in parte, ai dialetti dell'Italia settentrionale (alcune parlate venete, lombarde, piemontesi). Marchetti (1967) collega il loro impiego con la sottolineatura di una relazione di anteriorità fra due azioni aggiungendovi l'idea di occasionalità. Filzi (p. 62), già nel 1914 analizzando il fenomeno di bicomposizione nei dialetti dell'Italia settentrionale, gli ha attribuito soprattutto il valore aspettuale (la perfettività dell'azione). Quello che risulta dal mio corpus è la conferma delle conclusioni accettate dai due studiosi ed anche dalla maggioranza dei linguisti che oggigiorno svolgono delle ricerche nel campo della ladinistica.

6.1. La maggior parte degli esempi nelle due opere di Sgorlon testimoniano di una qualità peculiare dell'azione espressa da un tale sintagma. Si tratta frequentemente dell'azione che in un dato contesto rappresenta lo sconvolgimento del contenuto o un eco particolare. Gli esempi tipici ne sono il (24), il (25) ed il (26). Nel (24) l'uso del trapassato bicomposto dà all'azione una sfumatura sottile di solennità: l'eroe del romanzo era troppo forte e combattivo per chiunque fosse mai stato in grado di confrontarsi con lui. Nel (25) l'autore, raccontando gli avvenimenti della propria infanzia, si meraviglia del fatto che "l'Uomo con l'orologio d'oro" avesse deciso di venire proprio a casa loro. Si pone la domanda al condizionale passato bicomposto per esporre la misteriosità delle sue visite. "Lui, che veniva da lontano, doveva avere un motivo molto forte per le sue visite«, è il ragionamento del ragazzo. Certamente, il fanciullo conosceva bene la risposta poiché subito dopo dice: "Non potevo immaginarmi nessun altro motivo«. Il motivo delle sue visite era Jole, la mamma del narratore. In modo simile, nel (26) il condizionale passato bicomposto produce un effetto particolare, altrimenti lo scrittore avrebbe potuto usare semplicemente il condizionale passato, senza ricorrere ad un sintagma così complesso. Anche il passato prossimo bicomposto nel (27) dà un effetto solenne al messaggio: 
(27) [...], pre Luigi Zuliani (1876/1953) che al è stât pređi a Çurçuvint par ben 53 agns a dilunc. Prin come capelan e maestri (dal 1900 al 1905), e po come plevan, fintremai al 1953 cuant che al è vignût a mancjâ. Un afiet pardabon grant al à vût leât chest predi a la sô int, fondât su la scletece e sul rispiet, tant che il so ricuart inmò in dì di vuê al è vîf e presint. (P., Ot. 1997, 14)

Il brano è preso dall'articolo La buine int di Çurçuvint nel quale Renzo Balzan parla del prete che nel corso dei 53 anni di vita e del servizio religioso in questo piccolo paese della Carnia si è affezionato moltissimo agli abitanti del paese guadagnando la loro stima e fiducia. L'uso della forma bicomposta sembra dunque evidenziare il legame stretto fra il prete ed i suoi credenti.

Se ammettiamo come molto probabile la spiegazione basata sulla presenza del fattore temporale e di quello aspettuale, non possiamo al tempo stesso negare l'osservazione che in tutte le situazioni dal (22) al (27) esiste anche un motivo extralinguistico per l'impiego dei sintagmi detti bicomposti. Può darsi che il vero motivo per il loro uso sia la tendenza del parlante o dello scrittore di sottolineare più che l'azione stessa le circostanze inconsuete in cui l'azione è stata oppure sarà svolta.

6.2. Da notare il fatto che in alcuni esempi appaiono dopo la congiunzione "come se«, nelle proposizioni modali, come nell'esempio (23), ma anche nel

(28) Difat mi pareve di vê, des lez e des robis proibidis une sorte di idèe nassude cun mê, come se vie pal sium 'o vès vât viodût un agnul che mi ves fat segnos plens di autoritât par impedîmi di fâ alc: un di chei agnui ch' a comparivin tes Scrituris, ch' a ordenavin un sacrifizi o lu fermavin a colp, opûr ch' a fasevin nassi vocazions ch' a duravin dute la vite. (II dolfin, 31)

6.3. In alcuni altri casi li notiamo nelle proposizioni oggettive, ad esempio nel (22) o nel

(29) Ma 'o vevi pôre che il gjal al vès vût cjantât denant ch' o rivàs a sveâ Geneviève. (Il dolfin, 217)

6.4. Si trovano anche in qualche proposizione consecutiva:

(30) Ma il cûr di Jole al jere par chel âtri, adimplen, e cussì le veve vude vinzude il plui zovin. (Il dolfín, 162)

6.5. Non sono rari i casi dei timps bi-composci (Zof, 2000) nelle proposizioni relative introdotte da dulà che:

(31) Ma quanche si voltavisi indaûr, si visavisi che il bacìn dulà che si veve vût navigât fin a poc timp indaûr, e che su di lui si faseve tant stât, si jere sfantât. (Il dolfinn, 139)

(32) Si tratave di une cjase brusade e sdrumade, dulà ch' e veve vût stât une strie famose in dute la valade. (Il dolfin, 32)

6.6. L'esempio seguente dimostra l'uso del trapassato remoto bicomposto nella proposizione temporale:

(33) 'O cenarin cidins e po 'o lerin a durmî, dopo che Jole 'e vè vût controlât, minuziose, ogni scûr, ogni puarte, ogni clostri, ch' a fossin siarâz benon. (Il dolfin, 137) 
6.7. Nel (24), (27) e nel (34) che segue, viene adoperato il trapassato bicomposto o il passato bicomposto rispettivamente nelle proposizioni indipendenti:

(34) Mi vevin vât fevelât ancje des cigognis e des aganis di Gjarmanie. (Il dolfin, 169)

6.8. Nella rivista La Patrie dal Friûl ho trovato tre esempi dell'impiego del passato prossimo bicomposto nelle proposizioni principali che introducono il discorso diretto, in due di loro la VA è scrivi, in uno declarâ:

(35) ॠAl è segnâl ch' o vin di lâ indevant cul impegn di infuartî l' odontotecniche taliane, par fâle cressi in Europe, mantignint la nestre particularitât, venastai un sistem di impresis piçulis e minimis«, al à vût declarât Mestroni, daspò de sô conferme, plausade ancje dai sorestants de Union dai artesans dal Friûl. (P., Nov.1998, 7)

(36) Sul cont di Siro Angeli il critic, professôr Zorç Faggin, autôr cul professôr Walter Belardi da l'antologie "La poesia friulana del Novecento « al à vût scrit: "La discuvierte dal furlan tai agns Sessante no à mancjât di dâ bogns risultâts poetics, almancul tai câs che a an ubidît a une esigjence sintude, plui che a une mode dal moment. (P., Av.-Set.1999, 17)

(37) [...] Il critic e studiôs de leteradure furlane, professôr Zuanfranc D'Aronco, che de seconde edizion da »L'âga dal Tajament « al à vût scrit la jentrade, al marche cemût che: »Il lengaç al è net, intat. Un esempli di resistence di une fevelade locâl rispiet a la koinè de lenghe furlane, [...]«. (P., Av.-Set.1999, 17).

A questo punto aggiungo tre esempi trovati fuori dal corpus, nelle riviste Gnovis Pagjinis Furlanis e Sot la Nape, con le VA scrivi e dît, in testa sia del discorso diretto sia del discorso indiretto:

(38) Al dîs che cualchidun, une volte, al à vût scrit che la poesie furlane e je nassude intal 1945 oben - di ce ch' al somee - dome daspò de vuere. (GPF,1999, 17)

(39) E je vere che il procès di globalizazion al è plen di pericui, ma come che al à vût scrit Tullio De Mauro intune interviste tal nr.1/98 de riviste "Cinemasessanta《: "I procès di planetarizazion a dan la cussience de diversitât e de fuarce de diversitât etniche-culturâl a cualsisei ethnos, a cualsisei tradizion culturâl.»

(40) Ancje in te Biblie si lein cetantis cjossis sul cont dai siuns. Diu ur à vût dit a Marie e a Aron: "Scoltait lis mes peraulis! [...] ( (Sot la Nape, Gênar-Jugn 1996, 106)

Partendo dagli esempi (35) - (40) si può trarre la conclusione che gli esempi sporadici nelle riviste friulane contemporanee, quindi nel linguaggio giornalistico, dimostrano l'impiego dei tempi bicomposti nei verbi dichiarativi introducendo il discorso diretto ed il discorso indiretto. Delle ricerche più ampie sarebbero certamente necessarie per sostenere tale ipotesi.

6.9. In base al mio corpus è impossibile confrontare la situazione nel friulano con quella in francese poiché nella versione francese del Vangelo e nell'opera di SaintExupéry non vi sono esempi con le formes surcomposées. Dato che i testi letterari di origine friulana, tranne qualche breve racconto, finora non sono stati tradotti in francese, ma anche le versioni di opere letterarie francesi in friulano sono rarissime, le possibilità di svolgere tale ricerca sui testi moderni sembrano molto limitate. Di 
conseguenza, per fare i confronti nell'ambito della bicomposizione, devo ricorrere ai grammatici francesi Damourette e Pichon (1911-1936) ed allo svizzero Cornu (1953) che descrivono tuttavia la situazione in varie tappe dello sviluppo della lingua francese fino alla metà del ventesimo secolo. Secondo loro in francese prevale, benché sia meglio dire prevaleva, l'uso del passé surcomposé, cioè del passato prossimo bicomposto, mentre sono nella parte friulana del mio corpus più numerosi $\mathbf{i}$ casi del trapassato bicomposto e del congiuntivo trapassato bicomposto. Gli altri tempi bicomposti in francese compaiono esclusivamente nei testi letterari dei secoli passati. Fra gli esempi da loro citati predominano quelli in cui il passé surcomposé viene usato nelle temporali introdotte dalle congiunzioni come quand, après que, lorsque, dès que, e quelli nelle frasi semplici dove, accompagnato da un avverbio di modo o tempo, esso serve probabilmente a mettere in rilievo la compiutezza rapida dell'azione. Vi si trovano anche degli esempi nel periodo ipotetico, nelle relative, nonché nelle proposizioni indipendenti, ma non se ne trova traccia nell'introduzione del discorso diretto oppure indiretto come in friulano (vd.6.8.).

6.9.1. Carla Marcato (1986), a suo turno, analizzando lo stesso fenomeno nelle parlate venete e ladino-venete, finisce per constatare che la più frequente è la forma bicomposta relativa al passato prossimo (vd. C. Marcato, p. 50). La sua conclusione dunque concorda con la situazione in francese, opponendosi d'altra parte alla situazione in friulano. Neanche nella sua analisi si nota nessun esempio di tipo caratterizzato in 6.8., ciò che è del tutto logico tenendo in considerazione che non esistono riviste pubblicate in veneto.

\section{PERIFRASI VERBALI CON IL GERUNDIO}

Sotto questo titolo si classificano due PV: stâ+gerundio e lấ+gerundio (vd. 4.). Come già detto, entrambe sono portatrici del valore progressivo, cioè dimostrano l'azione nel processo del suo svolgimento.

7.1. Si sente tuttavia una sottile differenza nel loro carico semantico. Nel stâ+gerundio la componente di progressività sembra più marcata, mentre invece lo scrittore scegliendo lâ+gerundio mette in rilievo piuttosto l'azione stessa. »Lo scrittore«, dico, e non il parlante, poiché non ho mai sentito un friulanofono usare la variante con la CO lâ nel parlato. Siccome studio il friulano come lingua straniera, sebbene la mia ricerca concerna esclusivamente lo scritto, in certe occasioni non posso evitare di ricorrere alle testimonianze orali. Cerco delle informazioni da una parte dalle persone la cui lingua materna è il friulano, ma che a scuola hanno imparato soltanto l'italiano e che nella vita quotidiana usano le due lingue, dall' altra dai ricercatori che lavorano nell'ambito della friulanistica usando il friulano a livello universitario, cioè creando la lingua colta. Anche essi, avendo compiuto gli studi in italiano e comunicando soprattutto nell'ambiente linguistico italiano, nelle loro ricerche sul friulano, ragionano, benché senza esserne coscienti, sotto l'influsso dell'italiano. Ed a questo punto, la grande maggioranza dei friulanofoni a cui mi sono rivolto, o non 
conoscevano del tutto il sintagma lâ+gerundio, o hanno detto che non se ne servono nel parlato. Dal punto di vista del parlato esiste allora un dubbio a proposito della sua classificazione fra le PV. Anzi, la situazione nelle tre opere drammatiche incluse nel corpus confermano non solo la sua esistenza, ma anche la sua frequenza. Essa mi pare un'osservazione preziosa, però al tempo stesso ambigua. A giudicare dal fatto che di solito il linguaggio teatrale riflette abbastanza bene la realtà linguistica nella vita quotidiana, sarebbe logico dedurre che il sintagma in questione sia vivo anche nella lingua parlata. Ne offre alcuni esempi sporadici anche la rivista $\mathrm{La}$ Patrie dal Friûl, raramente si nota qualche esempio nella Bibie, al contrario del Piçul princip e dei romanzi di Sgorlon dove non viene usato. Può darsi che scrivendo opere drammatiche, invece di pensare in friulano, gli autori semplicemente trasformassero nel friulano il modello di pensare italiano. E lo stesso potrebbe valere per gli autori degli articoli nella rivista. Considerando il problema da questa ottica, la conclusione sarebbe che il sintagma rappresenti un calco sintattico sull'italiano dove andare+gerundio è una PV parzialmente grammaticalizzata, usata non solo nello scritto ma anche nel parlato.

7.1.1. In tutti gli esempi friulani raccolti nel presente corpus, tranne uno, la $\mathrm{CO}$ appare alla terza persona singolare (dell'indicativo presente, imperfetto e del futuro):

(41) Dele 'e reste inmòbil avilide sot de fasce de lûs mentri il tendòn al cale une vore adâsi e il cjant al va cressint. (Mich., 40)

(42) La lûs 'e va distudànsi a planc a planc intant che i CAVALîRS si jèmplin lis tazzis pal prìndis dal zurament. (Str., 55)

(43) $\mathrm{Al}$ è in preson a Glemone. Lu àn metût dentri par pulitiche, ch' al lave slengazzant il guviâr dal Patriarcje. (Buje, 31)

(44) »Il lengaç al è net, intât. Un esempli di resistence di une fevelade locâl rispiet a la koinè de lenghe furlane, che e va imponintsi tra i scritôrs e i poets di chenti, sigurementri cun vantaçs pratics, ma ancje cu la pierdite di tantis origjinalitâts e particularitâts, che a son, o ben che a jerin, la grande ricjece dal furlan«. (P., Av.-Set. 1999, 17)

(45) Sul cuel plui grant a' nàssin doi cjscjei maraveôs, la Glesie mari - che po 'e larà cuistant simpri gnovis oparis di art - e a' vegnin faz i Statûz e la loze de Cumune... (Buje, 81).

L'unico caso in cui notiamo la $\mathrm{CO}$ alla terza persona plurale rappresenta il

(46) [...] Za cumò, chei ch'a tègnin pai todescs, a' dìsin che i furlans, propite in chei dîs achì, a' pàssin di un paron sot di chel altri, lis cjàpin di chesc' e di chei, $a^{\prime}$ van piardint nemâi, implanz, racolz e cjasis e no san a ce sant avodâsi e, magari, a' sarà ancje vere...! (Str., 66)

7.1.2. Nel Vanseli seont Luche se ne trova un esempio nella forma interrogativa:

(47) Po ur disè: »Cemût mai si vadial disint che il Messie al è fî di David? « (Luche - 20,41).

7.1.3. Nel francese contemporaneo, il sintagma corrispondente aller +gerundio si usa raramente ed è esclusivamente una caratteristica della lingua scritta, malgrado un livello assai alto di grammaticalizzazione nella lingua classica. Contrariamente al 
friulano ed all'italiano, il francese dispone di un'altra variante del medesimo sintagma, aller en +gerundio, vale a dire tra la $\mathrm{CO}$ e la VA vi si può inserire l'elemento accessorio, la particella en. Grevisse (vd. p.1194, \& 790) vede la variante con la particella come più colta e secondo lui in questa variante aller manterrebbe un po'del suo valore di verbo di moto sebbene nella maggioranza dei casi questa sfumatura non si senta più, ed aggiunge che la variante senza en sembra più letteraria. Mettiamo a confronto i seguenti:

(48) "Son travail ira en s' améliorant «. (Dict. Larousse, 45)

(49) Une onde sonore qui allait s'élargissant (Grev., § 790, p. 1194, cit. CAMUS)

(50) Le chômage va augmentant. (Jereb, 125)

Accanto agli esempi con la $\mathrm{CO}$ nel presente, imperfetto e futuro, Grevisse ne cita anche uno con aller nel condizionale:

(51) Il suffisait de creuser [...] une sorte de canal qui [...] irait en s'approfondissant régulièrement (Grev., § 790, p. 1194, cit. M. TOURNIER)

E persino uno con aller nel passé composé:

(52) La pratique des sacrifices humains est allée s'amplifiant au Mexique. (Grev., § 790, p. 1194, cit. J. SOUSTELLE).

Il sintagma verbale nel (52) si vedrebbe certamente definito come una doppia PV, cioè come una giuntura perifrastica.

Siccome la parte francese del mio corpus non offre nemmeno un esempio di tale tipo, vengono citati qui sopra cinque esempi dalle grammátiche e dai vocabolari francesi.

7.2. D'altro lato, non sono in niente contestabili l'uso ed il valore della PV stâ+gerundio. La sua diffusione generale nell'uso corrente si riflette bene anche nel corpus dove non vi mancano esempi sempre negli stessi tre tempi del modo indicativo, cioè nel presente, imperfetto e futuro, però a differenza del sintagma analogo, lâ+gerundio, essa viene usata in tutte le persone sia del singolare che del plurale. Rispetto alla situazione in italiano dove la PV stare+gerundio si è stabilita come il mezzo principale per l'espressione di progressività e si vede completamente grammaticalizzata, la variante friulana si impiega comunque meno frequentemente perché la progressività vi si può esprimere in altri modi, soprattutto mediante le $\mathrm{PV}$ jessi daûr a+infinito e stâ a+infinito, nonché la locuzione verbale di tipo aperto con un elemento avverbiale, la indenant a+infinito, ovvero le PV, forse meno diffuse, lâ+gerundio (vd. 7.1.) e continuâ a+infinito.

7.2.1. Il francese a cui tale sintagma è ignoto si serve normalmente della PV être en train de+infinito. Quando invece si tratta dell'azione che si svolge nel periodo passato, in gran parte dei casi esaminati nel corpus il francese mette semplicemente il verbo di pieno significato all'imperfetto laddove in friulano viene usata la PV con il gerundio.

7.2.2. Confrontiamo prima un esempio estratto dal Piccolo Principe in tutte e tre le lingue: 
(53) fr. Je sentais bien qu' il se passait quelque chose d'extraordinaire. (Ex., 84)

frl. J capivi ch' al stave sucedint alc di fûr da l'ordenari.

it. Sentivo che stava succedendo qualche cosa di straordinario.

Va notato che la desinenza dell'imperfetto nell'originale francese ha la stessa funzione che le $\mathrm{CO}$ stave e stava rispettivamente in friulano ed in italiano.

7.2.3. Parlando della situazione in francese, bisogna tuttavia segnalare il sintagma arcaico être (en)+gerundio, oggidì fuori uso, con être al passé composé:

(54) La plupart des difficultés ont été s'aggravant, de saison en saison. (Grev., § 790, p.1194, cit. DUHAMEL).

Se ci rendiamo conto che il francese ignora il differenziamento stare $\leftrightarrow$ essere, vale a dire être vi svolge la funzione di ambedue, possiamo constatare che in un'epoca precedente nell'evoluzione delle lingue romanze il fenomeno di tale sintagma con il valore progressivo rappresentava una caratteristica comune tra l'italiano, il friulano ed il francese.

7.2.4. Ciò che d'altra parte sorprende è la prevalenza nelle opere drammatiche del mio corpus di due altri mezzi per l'espressione di progressività, stâ a+infinito e jessi daûr a+infinito, al posto di stâ+gerundio.

\section{PERIFRASI VERBALI CON IL PARTICIPIO PASSATO}

Vi fanno parte i sintagmi ormai definiti nelle grammatiche sotto il nome di tempi composti e quello di tempi bicomposti (esaminati in 5.4. e 6.), i sintagmi per l'espressione della voce passiva ed il sintagma lâ+part.pass..

Il presente lavoro non è dedicato in tale misura ai tempi composti quanto alle altre PV perché il mio obiettivo è soprattutto quello di esporre e prendere in esame la struttura sintattica ed i valori semantici di alcuni sintagmi friulani frequentemente usati che finora non sono stati esaminati in dettaglio o neanche esposti.

8.1. La mia osservazione è che i valori dei tempi composti nella maggioranza dei casi nelle due lingue sono quelli dell'espressione di anteriorità, perfettività e di posteriorità.

8.1.1. Vi sono delle particolarità per quanto concerne la scelta della $\mathrm{CO}$ jessi / être e vê / avoir. Il friulano può, secondo Faggin (1997, vd. p. 206), quando il lessema nella VA è un verbo pronominale, servirsi della $\mathrm{CO} v e \hat{e}$ invece di jessi, laddove il francese, come anche l'italiano, deve di regola utilizzare être:

(55) Par podê fâ dut chistu biel discors cul disen e cui colours, a coventava una persona dal mistêr e alora a àn cjatât la pitora Maria Ludovica Delendi, ch' a si à mituda a butâ jù cu la matita li' primis robis. (P., Av.-Set.1999, 8).

E' vero peraltro che il mio corpus offre degli esempi di questo tipo soltanto nella rivista, altrove si presenta in questi casi la $\mathrm{CO}$ jessi, conformemente al francese ed all'italiano:

(56) Alc si ere rot tal motôr. (Ex., 13)

(57) Al pareve ch' e si fos indurmidide, [...] (Pr.di s., 14) 
(58) Cussì al à vude remission dai nestris paris e si è visât de sô sante aleance, [...] (Luche - 1,72).

Siccome il punto di partenza della mia ricerca è la lingua scritta e non il parlato, dubito dell'autenticità di tale predominanza, poiché non è possibile escludere l'influsso dell'italiano.

8.1.2. Se mi allaccio anche questa volta al parere del Faggin (vd. p. 140), le VA bastâ, mancjâ, durâ richiedono, a differenza dell'italiano, la $\mathrm{CO}$ vê. Gli esempi del corpus non concordano sempre con la sua constatazione:

(59) Si consolave cul pinsîr che marz al jere za dongje, e dopo marz a' saressin bastâz i lens par fâ di mangjâ. (Pr.di s., 61)

(60) E il Tenent al veve reson đi jessi malfidant, la sô felicitât 'e jere durade dome pôs mês, [...] (Il dolfin, 162)

8.1.3. E' da notare la scelta di vê al contrario della situazione in italiano, ma in concordanza con quella in francese, nella giuntura perifrastica la cui CS dovût viene seguita dal verbo di motto lâ:

(61) Cuant che une grande part di nuâtris cjargnêi o vin scugnût lâ d'ogni bande pal mont, nissun si è preocupât di cjatâ soluzions par fânus restâ, [...] (P., Av.-Set.1999, 23).

"Siamo dovuti andare", sarebbe il sintagma corretto in italiano, mentre si dice in francese: "nous avons dû alleru.

8.2. Il friulano dispone di due $\mathrm{CO}$, jessi e vignî per la formazione delle $\mathrm{PV}$ con il valore di passivo. Riguardo a questo fenomeno il friulano e l'italiano sembrano essere in perfetta concordanza, contrariamente al francese il quale conosce una sola $\mathrm{CO}$, être, e segue dunque la propria via. L'esempio seguente, tratto dalla Bibbia, illustra bene la differenza tra il francese da un canto ed il friulano e l'italiano dall'altro:

(62) frl. Sicheduncje nô, rifletint cence vêl su la muse la glorie dal Signôr, o vignin trasformâz in chê stesse filusumie, di glorie in glorie, daûr de vore dal Signôr, ch'al è Spirt. (Sec.let. ai Cor. - 3,18)

it. E noi tutti, a viso scoperto, riflettendo come in uno specchio la gloria del Signore, veniamo trasformati in quella medesima immagine, di gloria in gloria, secondo l'azione dello Spirito del Signore.

fr. Et nous tous qui, le visage découvert, réfléchissons comme en un miroir la gloire du Seigneur, nous sommes transformés en cette même image, allant de gloire en gloire, comme de par le Seigneur, qui est esprit.

8.2.1. La CO vignî si può usare esclusivamente nei tempi semplici, cioè nell'indicativo e congiuntivo presente o imperfetto, nel futuro semplice, passato remoto e condizionale semplice. Nei tempi composti, dove si tratta infatti di giunture perifrastiche, la CO è sempre jessi e la CS stât, stade, stâz oppure stadis. L'esempio (63) dimostra una tale situazione, in cui la giuntura perifrastica in francese concorda interamente con quella in friulano ed in italiano:

(63) fr. Cet astéroïde n' a été aperçu qu'une fois au téléscope, en 1909, par un astronome turc. (Ex., 19) 
frl. Chest asteroid al é stât viodût di un astronim turc, cul telescop, une volte sole, dal 1909.

it. Questo asteroide $\grave{e}$ stato visto una sola volta al telescopio da un astronomo turco.

8.2.2. Maria Iliescu, nel suo lavoro Le frioulan à partir des dialectes parlés en Roumanie (1972), dice addirittura che jessi mostra tendenza a soppiantare vignî dappertutto, anche nei tempi semplici (vd. M. Iliescu, 3.3.8., p. 179). Faggin (vd. p. 205) e Zof (vd. p. 72), proprio al contrario, sostengono il parere che nei tempi semplici il friulano preferisce vignî.

In base all'analisi dei moltissimi esempi trovati nel corpus aderisco alla formulazione del Faggin e dello Zof. Tanti sono i casi in cui a prima vista la sequenza di jessi e del part.pass. rappresenti la PV per l'espressione del passivo, tuttavia, leggendo attentamente, si constata che in realtà il participio passato vi appare in funzione di aggettivo. In casi del genere jessi viene usato in qualità di verbo copulativo, quindi non possiamo definirlo come CO né parlare di PV.

In certi casi è comunque difficile porre dei limiti chiari tra la funzione verbale e quella aggettivale del participio passato. Per esempio, nei (64), (65), (66), (67), (68), non possiamo vedere un'azione ma uno stato, il participio passato vi ha indubbiamente la funzione di aggettivo:

(64) I prins libris a son scrits par latin-furlan, chei daspò a son scrits par venit-toscan. (P., Av.-Set.1999, 18)

(65) L'oficine, da tanc' àins 'e je siarade, inrusinide. (Mich., 33)

(66) E la tô creature no jere batiade. (Buje, 47)

(67) Un milion e 200 mil banderis par che dut il Friûl, de Livence al Timâf, ai 3 di Avrîl dal 1999 al sedi colorât di zâl e di blu, sot il simbul de acuile furlane. (P., Nov.1998, 5)

(68) [...] cemût ch' al jere vistût par solit chel lavorant. (Buje, 41).

Nei (69), (70), (71), (72), (73), al contrario, tratandosi dell'azione e non della descrizione dello stato, non si pone nessun dubbio sulla funzione verbale del participio passato, l'esistenza di PV è pertanto evidente:

(69) "Tal so sepulcri di Viene o larìn tal non e cul "spirt di Aquilee«, che il capucin al à rinovât sun chês stessis stradis d'Europe ch' a forin batudis dai missionaris de nestre Mariglesie, sul cricâ de evanzelizazion cristiane«. (P., Av.-Set.1999, 6)

(70) E vignît, dopo, a riferîmi quâi ch' a son chei che si rifudin di lâ in tai cjamps, ch' $a$ saràn cjapâz provedimenz. (Str., 77)

(71) In curt: o crôt di no falâ ritignint che buine part dal budget regjonâl al sedi doprât cence vêrs risultâts ma cuntune distribuzion acritiche e a ploie. (P., Nov. 1998, 19)

(72) Ch' e séi fate la Vuestre volontât, Pari santissin!« (Buje, 69)

(73). DEAN: E alore il bric ch' al scrivi: Petrussa, femine de Comunitât di Buje, ch' e à confessât di vê copade une sô creature, 'e je condanade a murî brusade in te sô cjase, in presinze dal popul. (Buje, 48). 
E'meno chiara la situazione nei seguenti:

(74) "La brute gnove nus à straneâts no pôc parcè ch' o jerin e $o$ sin impegnâts cul obietîf di liberâsi dai militârs«. (P., Nov. 1998, 6)

(75) Difat, fale une sole ecezion, l'Osservatori al é formât di personis che a àn competencis essenzialmentri glotologjichis e unevore scjarsis in altris cjamps. (P., Nov. 1998, 19)

(76) I fruts dal »timp plen« a van a scuele dal lunis al vinars par vot oris in dì, e in chestis oris a fasin dut il necessari par imparâ ce che al è indicât dai programs ministeriâi. (P., Ot.1997, 12)

(77) »Il rispiet e la promozion dal pluralisim a suponin ch' al sedi ricognossût il contribût des culturis e des lenghis regjonâls al nestri patrimoni nazionâl«. (P., Nov. 1998, 15).

8.2.3. Al contrario del (62), si nota persino qualche esempio sporadico nel quale la scelta della $\mathrm{CO}$ in francese corrisponde a quella in italiano, mentre la variante friulana si differenzia:

(78) fr. Et tu verras comme je suis bien obéi. (Ex., 40)

it. E vedrai come sarò ubbidito a puntino.

frl. E tu vedarâs cemût ch' $j$ vignarai ubidît.

Anche il (79) ci avverte che la concordanza fra il criterio di scelta della CO in friulano ed in italiano non è assoluta:

(79) frl. Perdonait e us vignarà perdonât. (Luche - 6,37)

it. [...] perdonate e vi sarà perdonato; [...].

8.3. La PV la $\hat{a}^{+}$part.pass., che corrisponde alla PV italiana completamente grammaticalizzata andare+part.pass., potrebbe in friulano essere considerata piuttosto come un italianismo. Se ne servono soprattutto le persone colte, probabilmente influenzate dall'italiano, la maggior parte dei friulanofoni che non studiano il friulano a livello universitario non l'usano nel parlato, certi nemmeno la conoscono. Se in italiano il sintagma esprime il valore di obbligatorietà, questo non significa automaticamente che tale sarebbe la sua funzione anche in friulano. Possiamo sostenere l'ipotesi che sia così, ma i parlanti friulani, per indicare l'obbligatorietà, di solito preferiscono adoperare altri mezzi, quali bisugne+infinito, vê di+infinito, scugnîtinfinito. Contrariamente alla lingua scritta che riflette un'immagine abbastanza differente. Nel corpus vi sono parecchi esempi dell'uso di lâ+part.pass., però essi compaiono tutti nelle opere drammatiche e nella rivista, eccetto uno che troviamo nello Sgorlon. Le grammatiche friulane non l'individuano, sicché è difficile decidere a che grado di grammaticalizzazione classificarla.

Basandosi sugli esempi dal corpus, non si può che confermare il suo valore ipotetizzato, cioè definirla come PV portatrice del valore obbligativo, almeno per quanto concerne la maggioranza dei casi. E' da dire che la CO si presenta sempre alla terza persona, sia singolare sia plurale, del tempo presente $\mathrm{o}$, raramente, dell'imperfetto: 
(80) Un blestemadôr, sar Pieri, ch' al va leât 'e berline par che il popul, ch' al è bielzà culì pront e parecjât, al puedi vergognâlu [...] (Buje, 25)

(81) Insumis, i lavôrs a' son di fâ e $a^{\prime}$ van fâz! [...] O sêso vô, che no vês voe? (Str., 77)

(82) Ma 'o jeri travanât di une scure, squasit dolorose sigurece che il paradîs nol jentrave par nuje. Il sclariment di dut al leve cirût cajù, su la tiare. (Il dolfin, 49)

(83) La cuistion dai inmigrâts, che e à inviât il contrast, e va studiade cun razionalitât e cûr. (P., Març 2000, 2).

Anzi, si notino nel corpus alcuni esempi nei quali la medesima PV, lâ+part.pass., marca ovviamente un altro contenuto grammaticale: la supposizione o la deduzione logica. Nell'

(84) Da Cormòns al Nadisòn al è lât brusât squasi dut [...] e chel che nol à ruinât il fûc, lu à ruinât il taramòt [...] e la peste, po, 'e à fat ancjemò di piês [...] (Str., 71)

dobbiamo in effetti riconoscere una giuntura perifrastica. Il suo equivalente italiano sarebbe deve esser stato bruciato ossia è andato bruciato. Ugualmente in

(85) I regiistris dai muarts a comencin dal 1650 cul volum cuart, chest al fâs pensâ ch' a sedin lâts pierdûts i prins trê volums. (P., Av.-Set. 1999, 18),

che tradurremmo in italiano: "I registri dei morti cominciano nel 1650 col quarto volume, e questo fa pensare che siano andati perduti i primi tre volumi«. Lo stesso valore si vede messo in evidenza nell'

(86) Pe Austrie-Ongjarie, difat, la cooperazion $e$ lave judade pal fat ch' e zove al svilup economic e no si veve pore di un svilup »nazionâl« di chestis formis imprenditoriâls, venastai a nivel di todescs, cecs, slovens, furlans e talians dal Imperi. (P., Nov. 1998, 7).

L'autore ci riferisce l'idea che »la cooperazione certamente aiutava«, cioè »doveva aiutare $\ll$.

8.3.1. Il francese, a differenza del friulano e dell'italiano, ignora tale sintagma, indicando il valore di obbligatorietà per mezzo di vari sintagmi con l'infinito: $i l$ faut + infinito, avoir à+infinito, devoir + infinito.

\section{PERIFRASI VERBALI CON L'INFINITO}

Le PV esaminate sotto questo titolo formano un gruppo più ampio che i sintagmi appartenenti ai due capitoli precedenti (vd. 7. e 8.).

9.1. Rappresentano l'unico mezzo per l'espressione dell'imperativo negativo in friulano le tre forme della PV con la CO stâ: no sta (a)+infinito, no stin a+infinito, no stait $a+$ infinito. La CO viene dunque preceduta dall'avverbio di negazione no e seguita dalla VA all'infinito. In questo caso parliamo di una PV completamente grammaticalizzata poiché il parlante o lo scrivente friulano non ha a disposizione nessun altro mezzo linguistico per l'espressione del medesimo valore.

9.1.1. Al plurale la $\mathrm{CO}$ e la VA vengono collegate indirettamente, mediante la preposizione a, mentre la seconda persona singolare sembra permettere sia il collegamento indiretto che diretto. Faggin (vd. p. 198) non vede ovviamente nessuna dif- 
ferenza fra "No sta a blestemâ!ı e "No sta blestemâlı, Zof non mostra nemmeno la possibilità di inserire la preposizione $a$ fra la $\mathrm{CO}$ e la VA, secondo lui si dice e si scrive $" N o$ sta fumâ!ı (vd. p. 74), "No sta lâ vie» (vd. p. 191). L'unica spiegazione logica dell'ellissi della preposizione $a$ alla seconda persona singolare sembra essere quella di evitare l'iato.

Il corpus riflette però l'immagine di una realtà diversa nel friulano scritto contemporaneo: non tutti gli scrittori tengono a questa regola. Se tutti e cinque esempi notati nel Pičul princip dimostrano che gli autori della sua versione friulana rispettano la regola di evitare l'iato, osserviamo proprio il contrario nei due romanzi di Sgorlon. Tanti casi di conservazione della preposizione vi testimoniano che l'autore non sente infatti nessun bisogno di ometterla:

(87) No sta fâle lungje, mi dà fastidi. Tu âs decidût di partî. Va mo. (Ex., 38)

(88) »No sta a esagerâ. Nol è sucedût nuje [...]« (Il dolfin, 212)

(89) »No sta a dî monadis, Ricardo.« (Pr.di s., 109).

Vi si trova tuttavia, dallo stesso autore, qualche esempio senza la preposizione:

(90) »Vonde, vonde, no sta strassâ lis tôs profeziis [...] (Il dolfin, 233).

Nel Vangelo secondo Luca il sintagma ha sempre la struttura ellittica:

(91) Apene che le viodè, il Signôr al sintì compassion di jê e j disè: "No sta vaîu. (Luche - 7,13).

Lelo Cjanton ed Alviero Negro usano coerentemente la variante con $a$, Michelutti invece resta fedele all'ellissi:

(92) No stâ a vê pore, Pieri! (Buje, 15)

(93) No stâ a pensâ, Nusse. No stâ a pensâ. Nissun al ûl fâj dal mâl a jê. (Str., 108)

(94) No sta cjòlij cheste pussibilitât umane, crodimi. (Mich., 21)

9.1.2. Un'altra osservazione: soltanto nelle opere teatrali Buje e Strumîrs e Zambarlàns la $\mathrm{CO}$ prende l'accento circonflesso, vale a dire appare veramente nella forma dell'infinito. Altrove, come possiamo vedere dagli esempi del corpus nonché nel Faggin e nello Zof (vd. 9.1.1.), va notata dappertutto la forma senza il circonflesso. Questo fatto ci porta a pensare che l'elemento sta secondo i due grammatici non si presenta in qualità di infinito, ma come forma di imperativo affermativo. Sull'elenco dei verbi analitici di Federico Vicario (1997, Appendice, p. 314) viene però citato un esempio nel quale l'autore usa la forma con l'accento, quindi l'infinito: no stâ rompi i tabars!. Abbiamo dunque il dilemma se il sintagma per l'espressione dell'imperativo negativo alla seconda persona singolare sia in realtà costituito dalla forma dell'imperativo affermativo oppure dall'infinito. Nel primo caso lo considereremmo una PV tipica, nel secondo atipica.

9.1.3. Un fenomeno straordinario che scopriamo nell'opera di Michelutti è l'apparizione della struttura ellittica non solo al singolare, ma perfino al plurale, forse per analogia:

(95) Bevìn e no stin pensà. (Mich., 18)

(96) No stàit dî cussì e dàjsi fuarce, ancje pe sûr. ' $O$ voi a viòdi di jê. (Mich., 34). 
9.1.4. All'interno della famiglia romanza il friulano rappresenta l'unica lingua in cui la norma richiede tali sintagmi per indicare l'imperativo negativo. Essi sono in parte conosciuti anche dai dialetti dell'Italia settentrionale, ne fanno uso soprattutto le parlate venete, ma sono sconosciuti nelle altre lingue e parlate romanze.

Il francese segue una via più semplice, mettendo il lessema verbale alla forma dell'imperativo affermativo e ponendolo fra due avverbi di negazione, non e pas. Confrontiamo l'esempio (87) con la variante originale di Saint-Exupéry:

fr. Ne traine pas comme ça, c'est agaçant. Tu as décidé de partir. Va-t'en.

Va ricordato a questo punto che la lingua parlata quotidiana conosce l'ellissi del primo elemento di negazione, ne. Sarebbe dunque del tutto normale dire »Traine pas comme ça [...]«.

Aggiungendovi ancora la versione italiana del medesimo enunciato (87),

it. "Non indugiare così, è irritante. Hai deciso di partire e allora vattene«., si può comunque rilevare un eventuale punto comune fra il sintagma friulano e quello usato in italiano letterario. Se accettiamo come più probabile la supposizione che nel sintagma "No sta fâle lungje", sta viene usato in qualità di infinito (vd. 9.1. sopra), possiamo constatare che entrambi i sintagmi contengono il verbo-lessema all'infinito.

9.1.5. Tali sintagmi si sono estesi però al di là dei confini del mondo romanzo. Sono penetrati come calchi sintattici nei dialetti sloveni occidentali dove si sono profondemente radicati. I sintagmi sloveni sono costruiti sul modello: avverbio di negazione[ne]oppure[na]+ imperativo affermativo del verbo stati + lessema verbale all'infinito. Skubic (1997), nel suo lavoro Romanske jezikovne prvine na zahodni slovenski jezikovni meji (Elementi linguistici romanzi nello sloveno occidentale), cita alcuni esempi di questo tipo e definisce i sintagmi in questione come una perifrasi verbale (vd. Skubic, 15.3., p. 95). Il significato originale del verbo sloveno stati, vale a dire »tenersi eretto, stare in piedi«, vi è già modificato, avendo perso il sema »eretto, in piedi«.

9.2. Particolarmente numerose sono in friulano le PV la cui $\mathrm{CO}$, seguita dalla VA all'infinito, è un verbo indicando la fase dell'azione. Ad esempio, stâ par+infinito, che svolge, come in italiano stare per+infinito, funzione imminenziale.

9.2.1. Ho ripreso il termine imminenziale dal de Castro (»State of Affairs«, »different Phasal Aspect distinctions«, p. 202) che nella sua descrizione dello spagnolo segue il modello di Dik. Quest'ultimo aveva elaborato per »lo stato di cose« in inglese uno schema in cui aveva proposto sette aspetti o gradi della medesima azione secondo la fase del suo svolgimento. Trasmettendo i sette gradi in friulano, li ho chiamati: 1. grât prospetîf 2 . grât prospetîf inmediât (= inminentiâl) 3 . grât ingresîf 4 . grât progresîf 5 . grât egresîf 6 . grât perfetîf inmediât 7 . grât perfetîf (= conclusîf). Come visto, la fase iniziale dell'azione in questa scala si divide in tre gradi: prospettivo, imminenziale e ingressivo. Il grado prospettivo immediato o imminenziale accenna un'azione avvicinante, un'azione sul punto di avvenire o di realizzarsi. 
9.2.2. La PV stâ par+infinito è stata classificata fra le PV parzialmente grammaticalizzate (vd. 4.). Secondo le testimonianze dei friulanofoni si usa frequentemente nel parlato. I testi inclusi nel corpus offrono esempi assai numerosi:

(97) E ve' che doi oms a vignirin a fevelâ cun lui: a jerin Mosè e Elie, comparîz te lôr glorie, e a fevelavin de sô partence ch' e stave par colmâsi a Gjerusalem. (Luche -9,31)

(98) Dopo di chesc' faz, il Signôr al sielgè âtris setantedoi dissepui e ju mandà doi par doi denant di sè, in ogni sitât e lûc ch'al stave par visitâ. (Luche - 10,1)

(99) Veglait e preait di un continuo, par vê la fuarce di scjampâ denant di duc' chesc' mâi ch' a stan par capitâ e par presentâsi denant dal Fî dal om. (Luche - 21,36)

(100) Chei ch'a jerin cun lui, apene che si inacuargerin di ce ch' al stave par sucedi, $\mathrm{j}$ diserin: "Signôr, vino di doprâ la spade?«. (Luche $-22,49$ ).

Dalle versioni francesi corrispondenti vediamo che vi appaiono due PV, aller+infinito e devoir+infinito:

fr. Et voici que deux hommes s'entretenaient avec lui: c'étaient Moïse et Elie qui, apparus dans la gloire, parlaient de son départ, qu' il allait accomplir à Jérusalem.

fr. Après cela, le Seigneur en désigna encore soixante-dix autres et les envoya deux par deux en avant de lui dans toutes les villes et localités où lui-même devait se rendre.

fr. Veillez donc et priez en tout temps, afin d'avoir la force d'échapper à tout ce qui doit arriver, et de paraittre avec assurance devant le Fils de l'homme.

fr. Voyant ce qu' il allait arriver, les compagnons de Jésus lui demandèrent: „Seigneur, faut-il frapper du glaive?«.

In questi casi devoirtinfinito ha il valore di probabilità, di annuncio del futuro. Aller+ infinito è una PV completamente grammaticalizzata, che serve all'espressione del »futuro vicino«. Tale PV sembra ignota ai parlanti ed agli scrittori friulani, benché sul Nuovo Pirona (vd. p. 495) possiamo leggere la definizione del sintagma lâtinfinito la quale evidenzia che, probabilmente soltanto la varietà goriziana del friulano, dispone dello stesso mezzo linguistico con il valore identico al sintagma francese:

Va deventa $=$ sta per diventare, diventerà; larès acquista $=$ acquisterebbe: Gurizza $v a$ deventâ un desèrt (Favetti 135) - Gurizza

va deventâ una vila (id.143) - Gurizza larès acquistâ un'entranza supiarba (id.144)

Vi segue il commento che il sintagma costruito allo stesso modo e portatore dell' identico valore non è »estraneo, sebbene non comune«, alle altre zone del Friuli.

Talvolta la CO nella PV stâ+infinito viene sostituita da jessi:

(101) Intant che lôr a scoltavin chestis robis, Gjesù ur contà ancje cheste parabule, parceche al jere donghe di Gjerusalem e lôr a crodevin che la manifestazion dal ream di Diu $e$ fos par rivâ. (Luche - 19,11).

Anche Faggin, nel capitolo Verbi servili e fraseologici (vd. p. 197) dà un esempio con la $\mathrm{CO}$ jessi che però traduce in italiano con stare e non essere:

- »'orloi al è par bati miegegnot, l'orologio sta per battere mezzanotte.«. 
9.2.3. Nel corpus si nota raramente il sintagma jessi sul pont di+infinito che corrisponde al francese être sur le point de+infinito ed all'italiano essere sul punto ditinfinito col carico semantico imminenziale:

(102) Ricardo al fo sul pont di domandâ: parvìe ch' o sês stât in preson?, e si stratignì juste in timp. (Pr.di s., 100).

9.2.4. Nel Grevisse (vd. $§ 791$, p. 1196) possiamo tuttavia leggere dell'esistenza del sintagma francese, ovviamente arcaico e oggidì non usuale, être pour+infinito, col valore di "futuro prossimo«, donde risulta che in un periodo passato dell'evoluzione delle lingue neolatine il friulano ed il francese conoscevano lo stesso sintagma il quale si è mantenuto in friulano fino al periodo contemporaneo, mentre si è quasi perso in francese.

9.3. Metisi a+infinito appartiene al gruppo di sintagmi verbali che stanno entrando nel processo di grammaticalizzazione, dunque ai cosiddetti casi limitrofi (vd. 4.). Il fatto che il primo elemento verbale, metisi, conservi il suo carico semantico originale quasi intatto, ci porta a credere che si tratti in realtà di una locuzione verbale e non di PV. Non possiamo contestare la prevalenza della dimensione semantica del sintagma su quella sintattica. Inoltre, se c'è $P V$, il secondo elemento verbale non è sostituibile dal sostantivo, ciò che non vale in questo caso. In una frase come »Pieri si met a lavorâ«, il carico semantico del lessema verbale all'infinito può ugualmente esser reso dal sostantivo vore, l'enunciato Pieri si met a vore comunica la stessa idea, da dove rileva che non c'è PV. D'altro canto è vero che il sintagma è talmente diffuso, almeno nella lingua scritta, da suggerire l'ipotesi che già vi si senta il processo di grammaticalizzazione. Giungiamo dunque alla conclusione che è il caso di una PV in via di grammaticalizzazione.

9.3.1. Metisi a+infinito copre in effetti doppia funzione: da una parte si usa come equivalente di stâ par+infinito (vd. 9.2.), preparâsi a+infinito, ossia prontâsi a+infinito, quindi per indicare la fase imminenziale dell'azione, d'altra parte con la marca della fase ingressiva, vale a dire per l'espressione dell'inizio nel senso proprio della parola. Paragonando il

(103) Nol podé dî nuje di plui. Si meté di colp a sangločâ. (Ex., 32)

col

(104) PIERI: Varèssio ancje di metimi a lei, cumò? (Buje, 17), vediamo che nel primo caso il sintagma segnala l'ingressività, l'inizio diretto dell'azione, dunque potrebbe venir sostituito dagli equivalenti (s)comenzâa a+infinito e tacâ $a^{+}$infinito, nel secondo caso invece esso marca una sfumatura di significato più vicina a quella di 'intraprendere un'attività lunga ed esigente', quindi parliamo piuttosto di una fase introduttiva, di uno stadio preparatorio, e non ancora del vero inizio dell'azione. Allora il (104), in cui metisi a+infinito fa parte di una giuntura perifrastica (varèssio di metimi a lei?), riflette il suo valore imminenziale. 
9.3.2. In alcuni casi, ad esempio nel (105), la sua funzione resta ambigua:

(105) Eliseo al veve fan. Al tirà fûr de valîs l'ultin toc di une pagnoche grande comprade a Napoli la sere prime, e al si metè a mangjâ. (Pr.di s., 15).

"Al si metè a mangiâ" si potrebbe interpretare sia 'al tacà a mangiâ' (ingressività) che 'si prontà a mangjầ' (imminenzialità).

9.3.3. Che si tratti di un valore o dell'altro, rari sono i casi dove il soggetto del metisi a+infinito non sia un essere vivente ovvero una cosa personificata. Il soggetto di solito decide coscientemente di intraprendere l'azione.

A differenza dei sintagmi affini tacâ a+infinito e (s)comenzâ $a+i n f i n i t o$, che sono in genere portatori del valore ingressivo. Il loro soggetto può essere animato nonché inanimato, di modo che l'azione non dipende tanto dalla decisione del soggetto, imponendo ovviamente al loro uso più automatismo e routine.

9.4. Dei tre sintagmi col valore ingressivo, tacâ $a+$ infinito è indubbiamente »il più friulano«. Né l'italiano né il francese conosce tale sintagma per specificare la fase iniziale nel senso proprio della parola. Nella maggioranza dei casi tacâ a+infinito sembra sostituibile dal(lo) (s)comenzâ a+infinito. Quest'ultimo, tuttavia, rimane limitato, in ambedue le varianti (con o senza il prefisso $-s$ ), soprattutto allo scritto, probabilmente sotto l'influsso dell'italiano.

Nel corpus tutti e due appaiono regolarmente, e come dimostrano gli esempi (106)-(111), senza differenza di carico semantico:

(106) ([...] Viodìnt che si inmaniè mâl 'e bat il cìmbli e 'a tache a cjantâ). (Str., 89)

(107) Dopo vei cjaminât òris, cidins, al vegnì gnot e lis stelis a tacârin a inpiâsi. (Ex., 79)

(108) Cuant tachino a esisti une civiltât e une storie furlanis? (P., Nov. 1998, 13)

(109) Aromai no j impuartave nuje che lu cognossessin, ma nol voleve che qualchidun lu fermas e al tacas a domandâj cemût ca e cemût là. (Pr.di s., 26)

(110) Jscomenčai a colp a viodi un tic di clâr tal misteri da sô presince e j domandai a sec: [...]. (Ex., 18)

(111) STUDIÂT: In ogni mût, chel al è il timp che Buje 'e scomenze a dâ figuris di rilêf... (Buje, 82).

9.4.1. Li ho classificati, come metisi a+infinito, fra i sintagmi entranti nel processo di grammaticalizzazione, poiché essi, a loro turno, possono a volte essere sostituiti dai sintagmi tacâ $a^{+}$[gruppo nominale] e (s)comenzâ $a^{+}$[gruppo nominale] rispettivamente. Per esempio, in una certa occasione l'enunciato 'E tache a fevelâ' equivale a 'E tache il so discors'.

9.4.2. Quando la $\mathrm{CO}$ si presenta all'imperfetto, non è comunque più possibile parlare dell'ingressività, l'inizio dell'azione è in tale caso visto come una fase durativa, un processo. Nel corpus sono stati notati alcuni esempi di questo tipo:

(112) J eri un grum in pinsîr par vie ch' $j$ comenćavi a nacuargimi che il dam al ere une vore grant, e l'aghe di bêvi ch' a lave al mancul mi faseve pensâ al pieis. (Ex., 29)

(113) Quanche il tramontan al scomenzave a businâ, ancje jo 'o stevi in scolt e mi pareve e no mi pareve di sintî un sunsûr di caroce ch' e coreve, e un sutîl sglinghinâ di cinglinais di cjaval. (Il dolfin, 26) 
(114) L' omp si jemplà la tazze e al tacave a bevi adasi adasi, ingrispant il cerneli come s' al cirìs alc tai siei ricuarz. (Pr.di s., 28).

L'identicità del fenomeno in francese è visibile dalla versione originale del (112):

$\mathrm{J}^{\prime}$ étais très soucieux car ma panne commençait de $m^{\prime}$ apparaittre comme très grave, et

l'eau à boire qui s' épuisait me faisait craindre le pire.

Da osservare la medesima situazione anche in italiano:

Ero preoccupato perché la mia panne cominciava ad apparirmi molto grave e l' acqua da bere che si consumava mi faceva temere il peggio.

9.4.3. Vi si notano anche dei casi dove, pur essendo la $\mathrm{CO}$ al presente, non è sicuro se la PV serva ad indicare l'ingressività o il processo dell'inizio dell'azione. Nel (115), preso dal Saint-Exupéry, si può spiegare la PV commencer à comprendre sia come un processo di comprensione nel suo svolgimento ovvero come la compiutezza di un processo di pensiero dalla quale risulta la consapevolezza di un fatto che era sconosciuto prima di questo processo:

(115) fr. Je commence à comprendre, dit le petit prince. Il y a une fleur... je crois qu' elle m' a apprivoisé... (Ex., 68)

frl. $J$ comenči a capt, dissal il pičul princip. A jé une rose j crôt ch' a mi vej dumiesteât...

it. "Comincio a capire«, disse il piccolo principe. "C' è un fiore... credo che $\mathrm{mi}$ abbia addomesticato «...

Sarebbe possibile interpretare quest' enunciato anche mediante un avverbio di tempo: "Ah! Adesso capisco...".

9.5. Mi pongo la domanda se esiste e qual è (in quanto esiste) la differenza tra le due varianti della PV con la CO comenzâ e scomenzâ rispettivamente (vd. 9.4.). Nella gran parte degli esempi trovati nella versione friulana del Piccolo principe va notato che per l'espressione dell'ingressività gli autori si servono del prefisso $s$, per l'indicazione della fase iniziale nella sua durata adoperano invece la forma senza prefisso. In qualche esempio tuttavia la variante senza $s$ - viene utilizzata come marca dell'ingressività pura.

Nelle opere di Sgorlon, talvolta si manifesta la forma prefissata anche come marca del carattere durativo di un'azione in fase iniziale (vd. 9.4.2., es. (113)).

9.6. Specificano la progressività parecchie PV e locuzioni verbali friulane con l'infinito, fra cui la più usuale è jessi daûr a+infinito (vd. 2., es. (8)). Essa è definita parzialmente grammaticalizzata, poiché la $\mathrm{CO}$ jessi mantiene in parte il suo carico semantico primitivo, cioè esprime lo stato. La VA non può mai venire sostituita, all'opposto dei sintagmi specificanti 1'ingressività (vd. 9.3., 9.4.1.), da un gruppo nominale. Ad esempio, in

(116) In chest moment, l'umanitât e je daûr a vivi, [...] un timp dulà che nol puès plui jessi

il popul a fâle cressi, ma a son i esperts che le progjetin. (P., Av.-Set. 1999, 21)

non si deve in nessun caso utilizzare la vite al posto di vivi. Un segno sicuro dunque che il sintagma va grammaticalizzandosi. Il primo elemento di collegamento, l'avver- 
bio daûr contiene il sema 'dietro, vicino', la sequenza jessi daûr quindi attualizza l'azione espressa dal lessema verbale nella VA. Tenendo in considerazione la spiegazione di Vicario (vd. 4.9., p. 169) che »i verbi analitici con l'avverbio daûr indicano una relazione allativa (avvicinamento) «, possiamo rilevare che jessi daûr a+infinito rende l'idea di 'seguire un'azione da vicino'.

La PV corrispondente in francese, être en train de+infinito, ha una struttura simile. Come la PV friulana, anche essa consiste nella CO être, la locuzione avverbiale en train de e la VA all'infinito. Nei confronti di quella friulana, la locuzione avverbiale francese dà comunque l'idea più chiara del movimento, del processo dell'azione. Etre en train de+infinito è, a suo turno, grammaticalizzata ad un livello abbastanza alto, non si deve per esempio sostituire il lessema verbale della VA con il gruppo nominale. In una frase come "Elle est en train de traduire un poème" (vd. Jereb, p. 125), sarebbe impossibile usare la traduction al posto di traduire.

9.6.1. Un'osservazione interessante: se in ambedue le lingue la preposizione $a$ si presenta come caratteristica dei sintagmi con l'infinito indicanti le fasi iniziali e continuativi dell'azione (la serie friulana include tacâ a+infinito, (s)comenzâ a+infinito, metisi $a+i n f i n i t o$, prontâsi a+infinito, preparâsi a+infinito, jessi daûr a+infinito, continuâ a+infinito, stâ $a+$ infinito, lâ indenant a+infinito, indurâsi a+infinito, restâ a+infinito; alla serie francese appartengono se préparer à+infinito, s'apprêter $\grave{a}+$ infinito, se mettre $\grave{a}+$ infinito, tendre $\grave{a}+$ infinito, se prendre $\grave{a}+$ infinito, se disposer $\grave{a}+$ infinito, commencer $\grave{a}+$ infinito (eccetto nel linguaggio letterario e nei casi in cui bisogna evitare l'iato dove à può venir sostituito đa de), continuer $\grave{a}+$ infinito, persévérer $\grave{a}+$ infinito, persister $\grave{a}+$ infinito, rester $\grave{a}+$ infinito, s'obstiner $\grave{a}+$ infinito, s'acharner $\grave{a}+$ infinito), il francese être en train de+infinito vi rappresenta una deviazione, vale a dire non si inserisce, a differenza del suo equivalente friulano, nella medesima serie. Il motivo di tale deviazione sarebbe l'analogia con gli altri sintagmi francesi costruiti secondo il modello 'verbo essere+preposizione indicante il luogo+sostantivo' (es. être sur le point de+infinito, être à la veille de+infinito).

9.6.2. Esiste d'altra parte in francese anche il sintagma être après (à)+infinito, avendo la struttura nonché il valore del tutto identici a quelli del friulano jessi daûr a+infinito, essa suona però molto arcaica. Dal 1986 la norma francese in effetti non ammette più il suo impiego. La variante senza $\grave{a}$, dunque être après + infinito si sarebbe conservata in alcune varietà dialettali (berrichon, franc-comtois), nel francoprovenzale e nel francese canadese. E'quindi il caso di un mezzo linguistico che sopravvive in una lingua romanza più conservativa, ma che è quasi sparito dall'altra lingua romanza la quale ha subìto le maggiori modificazioni nel mondo romanzo.

9.7. Un simile fenomeno rappresenta la coppia stâ a+infinito, être à+infinito. Appaiono ambedue come mezzi per l'espressione di progressività, il sintagma friulano pur essendo oggidì una PV parzialmente grammaticalizzata, quello francese resta la caratteristica della lingua letteraria. Grevisse ne cita un esempio dal Proust: "Seul dans ma chambre comme maintenant, pendant que tous les autres étaient à dîner." (Grev., 1196). 
In italiano lo stesso sintagma, stare a+infinito, è ovviamente meno diffuso che in friulano. Se in italiano, per attualizzare l'azione e darle un carattere continuativo, prevale l'uso di stare+gerundio, il friulano scritto ricorre frequentemente ad entrambi, stâ a+infinito e stâ+gerundio, ed è difficile dire quale dei due prevalga. Nel Vangelo secondo Luca si trova un solo esempio in cui il friulano e l'italiano si servono dello stesso mezzo d'espressione:

(117) Frl Il popul al stave a cjalâ. (Luche - 23,35)

It. Il popolo stava a vedere, [...].

Il francese, in questo luogo, mette i due verbi alla forma personale collegandoli con la congiunzione coordinativa et:

Fr. Le peuple restait là et regardait.

9.7.1. Di solito, questo vale per il Vangelo e per l'opera di Saint-Exupéry, in friulano si osserva stâ a+infinito lì dove in francese viene usato solo il verbo di pieno significato:

(118) Frl. Sô sûr, ch' e veve non Marie, si sentà dongje dai pîs dal Signôr e a stave a scoltâ ce ch' al diseve. (Luche - 10,39)

Fr. Celle-ci avait une sœur appelée Marie, qui, s'étant assise aux pieds du Seigneur, écoutait sa parole.

(119) Fr. Si tu aimes une fleur qui se trouve dans une étoile, c'est doux, la nuit, de regarder le ciel. (Ex., 86)

Frl. Si tu tu ûs ben a une rose ch'a sta sore une stele, al é biel, di gnot, stâ a cjalâ il cîl.

Potremmo dedurre che i friulanofoni sentono un bisogno più forte di sottolineatura dell'attualizzazione dell'azione, perciò si servono della PV nelle situazioni dove per i francofoni un mezzo semplice rende l'enunciato pienamente chiaro.

Questa divergenza fra il francese ed il friulano mi porta a credere che l'uso della $\mathrm{PV}$ in friulano possa essere un pleonasmo, visto soprattutto che la lingua friulana dimostra una tendenza generale all'uso delle forme perifrastiche.

9.7.2. Lo Sgorlon e gli autori delle opere drammatiche incluse nel corpus utilizzano stâ a+infinito assai frequentemente, ma pochi esempi del suo impiego sono notati nella rivista:

(120) ' $O$ stèi a cjalâle fin che no fo disparude daûr di une crete. (Il dolfin, 270)

(121) ' $O$ starin a sintî ce che nus dìsin i ciapitanis ch'o vin clamât [...] (Str., 72)

(122) Eliseo al stave a sintî i discors senze mai viarzi bocje. (Pr.di s., 94)

(123) Eco. Al è inutil di stâ a dâti òrdins che tant tu fâs di to cjâf l'istès. (Str., 15)

(124) VIELI: Lì, al jere sepulît un gjenerâl roman, no sai di ce uere ... Fin che la sô cinise 'e jere lì dentri, a stavin a fâj la uardie magnis e sarpinz. (Buje, 57)

(125) Di fat il stes non di Merêt al ven fûr dal plui antic non di Mellereti, che al stâ a testimoneâ la vocatsion di chest teritori par la coltivatsion dal miluç. (P., Ot. 1997, 12)

Va notato però nella rivista, ciò che avviene rarissimamente, un enunciato nel quale la $\mathrm{CO}$ stâ appare in un tempo composto, al passato prossimo, quindi si tratta della PV atipica all'interno di una giuntura perifrastica: 
(126) Par savê alc di plui in proposit, $o$ vin stât a interpelâ la pressidente dal Comitât, che e je la siore Linde Cisilin di Vilevuarbe. (P., Nov. 1998, 3)

L'esempio è comunque un po' ambiguo: può darsi che in realtà non si tratti del valore progressivo perché stât sia usato nel significato 'lât', cioè per esprimere il moto verso un luogo (= o sin lâts a interpelâ), e in questo caso non si potrebbe parlare di PV, ma di locuzione verbale di tipo aperto (vd. 3.)

9.8. Faggin (vd. p. 198) parla, sempre in merito all'indicazione dell'aspetto progressivo, anche del sintagma jessi che+indicativo. Non pochi friulanofoni mi hanno confermato l'esistenza di questo mezzo linguistico dicendo che esso si sente nell'uso quotidiano, ma il corpus non ne offre nemmeno un esempio.

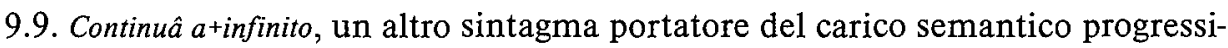
vo, suona meno friulano che jessi daûr a+infinito e stâ a+infinito. Si tratta ovviamente di un neologismo che appare comunque spesso negli articoli della Patrie dal Friûl, probabilmente sotto l'influsso dell'italiano. Dato che il primo elemento verbale conserva il suo significato originale e che in certi esempi il secondo elemento verbale può venire sostituito da un gruppo nominale, esso rappresenta, come le PV indicanti l'ingressività (vd. 9.3., 9.4.1.), un caso limitrofo, cioè parliamo di un sintagma entrante nel processo di grammaticalizzazione, quindi di una PV del terzo grado secondo il livello di grammaticalizzazione (vd. 4.).

Possiamo anche trovare qualche esempio sporadico nello Sgorlon, nonché nelle opere drammatiche, come nel caso seguente dove continua a+infinito fa parte della giuntura di tipo locuzione verbale $1+\underline{\mathrm{PV}}+\underline{\text { locuzione verbale2: }}$

(127) SANDRI No si pô, no (al pense). E, lassàle continuâ a tormentâsi a cirî? Podìno? (Mich., 39).

9.9.1. E'interessante il (128), tratto dal Piccolo principe, in cui il sintagma usato dal Saint-Exupéry poursuivre+gruppo nominale è stato tradotto in italiano con continuare+ gruppo nominale mentre i traduttori in friulano hanno scelto la PV continuâ a+infinito:

(128) Fr. Je poursuivis ma marche vers le mur. (Ex., 83)

It. Continuai il mio cammino verso il muro.

Frl. $J$ continuai a cjaminâ viers il mûr.

Mi pongo a questo punto la domanda perché non hanno utilizzato il gruppo nominale il cjamin. Nonostante avessero potuto seguire lo stesso modello che il traduttore dal francese in italiano e rendere così la traduzione più vicina all'originale, la loro preferenza era l'impiego della PV.

9.10. Discutendo le PV segnalanti il valore di progressività, dobbiamo individuare il sintagma lâ indenant a+infinito, che malgrado tanti scrupoli va classificato fra le locuzioni verbali. Vale a dire che, per quanto riguarda la struttura, il sintagma dimostra la concordanza quasi perfetta con la PV parzialmente grammaticalizzata jessi daûr $a+$ infinito, benché il significato dell'elemento avverbiale indenant sia proprio contrario a quello di daûr. Insomma entrambi gli avverbi producono nei due sintagmi lo stesso effetto. Dal punto di vista della struttura non si dovrebbe dunque esitare a conside- 
rarlo una PV, la sua diffusione invece non è tale che abbia acquistato funzione grammaticale. Nel parlato vi sembrano più frequenti i sintagmi lâ indenant cun+gruppo nominale e lâ indenant+avverbio di modo. Il corpus dispone di pochi esempi con lâ indenant a+infinito ed essi si notano soltanto nella rivista e nello Sgorlon:

(129) Se un popul al à di lâ indenant a tabaiâ une lenghe in maniere dignitose, al à di vê i vocabui juscj par fevelâle. (P., Av.-Set.1999, 21)

(130) Lui al veve dit di sì, ma al lave indenant a cusîsi di bessôl. (Pr.di s., 96).

9.11. Nel corpus vanno notati ancora due mezzi linguistici che servono ad esprimere il carattere progressivo dell'azione, sebbene più raramente: le locuzioni verbali restâ a+infinito e pratindi di+infinito. Il primo rappresenta l'equivalente del francese rester $\grave{a}+$ infinito, il secondo non ha pari in francese né in italiano:

(131) Dome che Bossi, secont Salvi, nol capis masse di culture e di lenghe e al pratint di slargjâ la Padanie a la Toscane e al Friûl che di Padanie no àn nuie. (P., Nov. 1998, 17).

9.12. Per rendere l'idea di egressività il friulano dispone delle PV finî di+infinito e fermâ di + infinito. Visto il loro uso frequente, entrambe stanno entrando nel processo di grammaticalizzazione, sebbene le $\mathrm{CO}$ mantengano il valore semantico originale e le VA siano a volte sostituibili dal gruppo nominale (vd. 4.).

$\mathrm{Nel}$ corpus finî di+infinito prevale e nella maggioranza dei casi la $\mathrm{CO}$ appare in un tempo passato, sia semplice che composto, cioè essa si presenta spesso come una PV atipica all'interno di una giuntura perifrastica:

(132) Ma il pičul princip, ch' al veve finit di parecjâsi, nol volé fâ patî il vecjo sovran: [...] (Ex., 43)

(133) Al fini di bevi ancje il vin, e po al lè dongje il banc. (Pr.di s., 17)

(134) Al è finît di cori il sanc, sì, ma... no lis lagrimis. Lis plàis, e' saràn lungis di vuarì. (Mich., 27)

Al contrario di fermâ di $i$ infinito la cui $\mathrm{CO}$ è limitata ai tempi semplici:

(135) Ma la plantute a fermà subìt di cressi e a comenčà a preparâ une rose. (Ex., 33)

(136) ROMANS: $a^{\prime}$ fermin di segnâ il pas e a' restin fêrs come statuis. (Buje, 14).

9.12.1. Quando la CO finî o fermâ si presenta all'imperfetto, la PV non è portatrice del valore egressivo nel senso proprio, vale a dire non si tratta di specificare il punto finale dell'azione, ma la fine dell'azione è vista come un processo nel suo svolgimento (cfr. 9.4.2.):

(137) Si sentà sul cjaruz, cu lis gjambis a pendolon, e quan'che il cjan al fermave di uacâ al sintive lis vôs e i sunsûrs de cene dentri cjase. (Pr.di s., 55)

(138) Il pičul princip, ch'al vedeve vignî indenant un butul unevore grant, al sintive dentri di sé ch'al sarés vignût fûr alc di meraculôs, ma la rose no finive plui di prontâsi par sei biele, scuindude tal so vuluč vert. (Ex., 33).

9.12.2. Nel romanzo Prime di sere e nell'opera teatrale Buje sono da notare i casi in cui si intromette la particella pronominale la oppure $l e$ col valore neutrale all'interno della PV finî di+infinito: 
(139) PIERI: cun fote Sint po, tu la finirâs di rompi [...] Jo no fâs nuje, e vualtris fasêt ce ch'o olês! (Buje, 17)

(140) »[...] E' finissile di porconâ, in presinze dal frut. « [...] Chei altris operaris si jerin tirâz dongje e ancje lôr j disevin che la finis di fâ tant bordel, che la colpe 'e jere ancje sô. (Pr.di s., 105-106).

9.13. Raramente possiamo notare nel corpus anche il sintagma smeti di+infinito, portatore del carico semantico egressivo:

(141) Tu no tu mi às dade la bussade, cu la cuâl che jê, di cuanche o soi chi, no à smetût di bussâmi i pîs. (Luche - 7,45).

Sebbene elencato nel Nuovo Pirona (vd. p. 1058), esso rappresenta probabilmente un calco sull'italiano.

9.14. I sintagmi corrispondenti in francese finir de+infinito, cesser de+infinito, (s')arrêter de+infinito, rappresentano ugualmente dei casi limitrofi (vd. 4.). Talvolta vi si adoperano perfino achever de+infinito e terminer de+infinito. Così per esempio nelle varianti originali dei (132), (135), (138), presi dal Piccolo Principe, notiamo i sintagmi achever+gruppo nominale, cesser de+infinito, finir de+infinito:

- Mais le petit prince, ayant achevé ses préparatifs, ne voulut point peiner le vieux monarque: [...]

- Mais l'arbuste cessa vite de croître, et commença de préparer une fleur.

- Le petit prince, qui assistait à l'installation d'un bouton énorme, sentait bien qu'il en sortirait une apparition miraculeuse, mais la fleur n'en finissait pas de se préparer à être belle, à l'abri de sa chambre verte.

9.15. E'di largo uso, nello stile scritto nonché nel parlato, il sintagma friulano rivâ a+infinito. I friulanofoni lo impiegano regolarmente per indicare il conseguimento dello scopo. Il carico semantico originale del primo elemento verbale, rivâ, si è già oscurato a tal punto che possiamo definirlo $\mathrm{CO}$, esso ha perso il sema »moto servando quello di »al punto finale«. Al tempo stesso ottiene il sema »l'azione«, cioè non è più il caso di 'portare se stesso al punto finale', ma di 'portare la sua azione al punto finale'. Inoltre, il lessema verbale all'infinito non è in nessun modo sostituibile dal gruppo nominale, si tratta quindi di pura VA. Ne risulta che il sintagma in questione si può classificare fra le PV parzialmente grammaticalizzate (vd. 4.). Vicario (vd. p. 304) segnala il suo impiego, assegna però molto più spazio all'altro sintagma portatore del medesimo carico semantico, rivâ adore di+infinito (vd. p. 144). Il corpus, all'opposto, dimostra solo dei casi sporadici di quest'ultimo, vi prevalgono gli esempi con rivâ a+infinito:

(142) Di jê si passionarin sîs zovins, che $\mathrm{j}$ levin simpri daûr, sperant di rivâ a otignî il so amôr, ma jê ju parave vie cun malegracie, ur sbeleave, ju sburtave-vie ridint, quanch'a vignivin masse dongje. (Il dolfin, 16)

(143) ANE Oh, Diu! Il to dovê...! Ma rivarino mai, Aldari, a jèssi omp e femine, in pâs, nô doi? (Str., 109) 
(144) 'O vevi tante robe di lavâ e no abadavi al frut. Di un moment a chel altri un cìul mi fâs drezzâ i cjavei sul cjâf: il frut al jere colât, l'aghe lu puartave vie. Vaint, mi buti dentri e'o rivi a puartâlu a salvament. (Buje, 68)

(145) La mari e i fradis a lerin une dì a cjatâlu, ma no rivavin a lâj dongje parvie de masse int. (Luche - 8,19).

Il sintagma identico, sebbene meno usuale, esiste in francese, arriver à+infinito, tuttavia, gli enunciati presi dalle varianti francesi del Vangelo e dell'opera di SaintExupéry non attestano la sua presenza. Nei contesti dove nella variante friulana viene usato rivâ a+infinito, in francese notiamo sia réussir à+infinito, pouvoir + infinito, savoir+infinito oppure parvenir à+infinito, ed in un assai grande numero di casi vi appare solo il verbo di pieno significato:

(146) Fr. [...] elles ne parviennent pas à s'imaginer cette maison. (Ex., 20)

Frl. [...] a no rivin a figurâsi cheste cjase.

(147) Fr. Je fus surpris de comprendre soudain ce mystérieux rayonnement du sable. (Ex., 78)

Frl. J mi maraveai di rivâ a capî dut un colp ce ch'al ere chel mistereôs slusorâ dal savalon.

(148) Frl. [...] alore lu peavin cu lis cjadenis e lu vuardeavin cui čocs, ma lui al rivave a crevâ dutis lis peandis e il diaul t'al sburtave tai puesc' plui deserz.

(Luche - 8,29)

Fr. [...] on le liait alors, pour le garder, avec des chaînes et des entraves, mais il brisait les liens et le démon l'entraînait vers les solitudes.

$E^{\prime}$ evidente in base agli esempi di questo tipo la tendenza del friulano all'utilizzo della PV lì dove il francese (e anche l'italiano) impiega il sintagma semplice che consiste in un solo elemento verbale.

9.15.1. Il francese arriver à+infinito è una caratteristica della lingua parlata e si usa di solito con la $\mathrm{CO}$ in un tempo semplice, nelle frasi come per esempio »Je n'arrive pas à comprendre«. Se per caso viene usato all'interno di una giuntura perifrastica, dunque se arriver si presenta al passé composé, non ci sarà dubbio sulla scelta della $\mathrm{CO}$ : la $\mathrm{CO}$ sarà être e non avoir. Allora si dirà, benché non sia abituale, "Je ne suis pas arrivé à comprendre«. Al contrario della norma friulana, la quale permette le due possibilità, jessi e vê (vd. 8.1.1.) Gli esempi nel corpus riflettono bene questa libertà di scelta, anche all'interno della stessa opera talvolta viene usata una talvolta l'altra CO:

(149) Chest al è il miôr ritrat che, plui tart, $j$ ài rivât a fâ di lui. (Ex., 14)

(150) J faséi capî al pičul princip che i baobabs a no son sterps, ma arbui granc' come glêsis e che, se ancje al ves puartât cun sé un trop di elefants, chest trop nol varés rivât a finî fûr un sôl baobab. (Ex., 24)

(151) J vegnivi propit a disj che, cuant ch' $\mathrm{j}$ no speravi plui, $j$ eri rivât a finî il gno lavôr! (Ex., 86)

(152) Disèimi, invezzit: sêso rivât a timp a fâj l'imbassade al cjastalt di Cjarisà? (Str.,13) 
(153) Il sindic di Triest, fintremai a vuê, al à rivât a imponi i interes de sô citât. (P., Nov. 1998, 10)

(154) E simpri o pensi che se une scuele come cheste e vîf e e crès intun piçul paîs, $o$ sin rivâts a preparâ alc di biel pal doman. (P., Ot. 1997, 12)

(155) Capitade la montane, il riu si è butât cun viamence cuintri di chê cjase, ma nol à rivât a movile, parceche e jere fate ben. (Luche - 6,48)

(156) 'O scomenzai a meti-vie bêz, ma dopo mês 'o jeri rivât a ingrumâ pôc e nuje. (Il dolfin, 153).

9.16. Tornâ a+infinito non rappresenta l'unico, ma certamente il più usuale mezzo linguistico friulano per l'espressione dell'aspetto iterativo o reiterativo. Se il fenomeno di iterazione indica una sola ripetizione, quello di reiterazione si riferisce a una seconda ripetizione oppure ripetizione costante della stessa azione. La lingua contemporanea dispone di un secondo mezzo, più semplice, la prefissazione. Si tratta di due prefissi, $r i$ - e re-, che sono probabilmente italianismi, poiché la lingua parlata non se ne serve. Esiste tuttavia un altro modo, più friulano, di esprimere la ripetizione dell'azione: aggiungere al verbo di pieno significato l'avverbio modificatore di gnûf $\mathrm{O}$ ancjemò une volte (vd. 4.). In seguito, non si può definire tornâ a+infinito una PV completamente, ma parzialmente grammaticalizzata.

Nel (157) essa è portatrice del valore iterativo, mentre nel (158) indica la reiterazione:

(157) 'L'astrònim al tornà a fâ la sô dimostrassion dal 1920 vistît dut elegant. (Ex., 21)

(158) 'Dut al jere efiet dal mût seren e precîs di contâ dal Pelegrin, che si compenetrave tai faz, e come in sium al tornave a vivi i avenimenz dal Vanzeli, e al fevelave di ogni robe cun tune precision plene di suns, di odôrs e di colôrs. (Il dolfin, 74).

Quando la CO si presenta all'imperfetto, non possiamo parlare che del valore reiterativo, il quale si avvicina però a quello progressivo, in effetti la distinzione fra ripetizione durevole e svolgimento continuativo è molto sottile.

Il francese indica i due valori mediante il prefisso $r(e)$-. Confrontiamo allora la variante friulana del (157) con l'originale:

Fr. L'astronome refit sa démonstration en 1920 , dans un habit très élégant.

9.16.1. In ambedue le lingue va notato, in alcuni esempi, il fenomeno di doppia espressione della reiterazione, cioè la $\mathrm{PV}$ o il prefisso rispettivamente, è seguito dall'avverbio modificatore:

(159) Fr. Je refis donc encore mon dessin: [...] (Ex., 14)

Frl. $J$ tornai a fâ ancjmò une volte il gno disén: [...].

A prima vista si potrebbe considerare il doppio mezzo per l'espressione dello stesso valore come un pleonasmo, però in realtà la sottolineatura di una seconda ripetizione diventa più forte in questa maniera. Dalla variante italiana si può vedere senza leggere il contesto che si tratta di reiterazione:

It. Rifeci il disegno una terza volta, [...]. 
9.16.2. Certi verbi francesi formati con re-che hanno acquistato il pieno significato ed in cui non si sente più la funzione grammaticale del prefisso devono essere tradotti in friulano mediante la PV tornâ a+infinito, tali: répéter $\rightarrow$ tornâ a dît, répliquer $\rightarrow$ tornâ a dî̀, réclamer $\rightarrow$ tornâ a domandâ indaûr, renvoyer $\rightarrow$ tornâ a mandâ (indaûr).

9.16.3. Per quanto riguarda la scelta della $\mathrm{CO}$ nella formazione dei tempi composti all'interno della giuntura perifrastica, vale a dire quando tornâ a+infinito si presenta come PV atipica, è da notare una situazione paragonabile a quella di rivâ a+infinito (vd. 9.15.1.). In base all'analisi della maggior parte degli esempi dal corpus possiamo concludere che la scelta dipende dal lessema verbale nella VA. Ad esempio, nel (160), sembra evidente che la VA comparî richiede la CO jessi:

(160) »Al é tornât a comparî Elie«. (Luche - 9,8).

Il comportamento del francese reparaitre concorda:

- „C'est Ëlie qui est reparu«.

La CO jessi nel (161), a suo turno, si orienta secondo la VA nassi:

(161) Mai plui però 'e sarès tornade a nassi tra di nô l'armonìe di amîs simpri insieme, che un al pareve l'ombrene di chel âtri. (Il dolfìn, 236).

Visto la VA inpiâ nel seguente, l'impiego di vê sembra logico:

(162) Ma parcé àstu tornât a inpiâlu? (Ex., 52).

E'meno logico però, sebbene la norma ammetta entrambe le $\mathrm{CO}$, che dal medesimo autore, in due esempi con la VA cjapâ tratti da due romanzi diversi, nel primo caso viene usato vê e nel secondo jessi:

${ }^{(163)^{~}}{ }^{E} E$ veve tornât a cjapâ-sù il lavôr di sartore, ma nol jere stât fazzil tirâ dongje une clientele in tun paîs che nol jere il so. (Pr.di s., 111)

(164) Forsit s' o fos tornât a cjapâ in man i segnâi, un par un, mi saressin parûz diviars di une volte, senze forme, senze significât, senze une vere indicazion. (Il dolfin, 217).

Forse la spiegazione risiede nel fatto che le opere di Sgorlon, prima della publicazione, sono state riviste dal professor Giorgio Faggin.

9.16.4. Sono interessanti due esempi dallo stesso articolo della rivista Patrie dal Friûl, dove appare una volta la PV atipica tornâ a viodi e l'altra volta il neologismo revisionâ:

(165) Dute la toponomastiche dal Comun e je stade tornade a viodi recuperant i toponims e i microtoponims origjinaris, no dome pai paîs, ma ancje pes stradis e pai lûcs. (P., març 2000, 6)

(166) [...] Ancje chenti la toponomastiche e je stade revisionade tirant fûr i nons origjinaris.

9.17. Usâ a+infinito si adopera in friulano per indicare un'azione abituale. Il sintagma rappresenta un caso limitrofo fra la locuzione verbale e la PV, passando gradatamente da un semplice modo di dire ad un sintagma col valore modale (vd. 4.):

(167) Ma, come ch' al usave a dî, »No si sa mai!« (Ex., 36). 
Il francese, che non dispone, per l'indicazione dell'abitudine, di un mezzo linguistico del genere, ricorre normalmente all'uso dell'imperfetto, come notiamo nella variante originale del (167):

- Mais, comme il disait: »On ne sait jamais!ณ.

\section{CONCLUSIONE}

La ricerca ha confermato il postulato iniziale che in friulano letterario contemporaneo la PV rappresenta una categoria grammaticale a parte o almeno una categoria in via di grammaticalizzazione. Vi si notano certi fenomeni paralleli al francese scritto contemporaneo, ma anche numerose divergenze. Per esempio, alcuni sintagmi esistenti in entrambe le lingue stanno sparendo dal francese contemporaneo, mentre nel friulano conoscono un'espansione. In base al corpus si può constatare che spesso laddove nel testo friulano appare la PV, nella variante francese si presenta il sintagma verbale più semplice il quale consiste solo in un lessema verbale.

Scrivendo in friulano, gli scrittori ed i giornalisti non sono sempre in grado di distinguere se tale sintagma sia davvero »friulano«, di conseguenza nei testi scritti si possono trovare calchi sintattici sull'italiano - sintagmi che in italiano rappresentano le PV grammaticalizzate, però in quanto sono ignoti al friulano parlato, non è opportuno classificarli fra le PV friulane. 


\section{Corpus}

(Ex.) Saint-Éxupéry, A. (1943) Le Petit Prince. Paris, Éditions Gallimard.

(Luche) Bellina, A. (1999) La Bibie: Il Vanseli seont Luche. Bologna, Grafiche Dehoniane.

(Pr.di s.) Sgorlon, C. (1975) Prime di sere. Udine, Societât Filologiche Furlane.

(Il dolfin) Sgorlon, C. (1993) Il dolfin. Tricesimo, Roberto Vattori Editore.

(Buje) Cjanton, L. / Negro, A. (1971) Buje. Udine, Societât Filologjiche Furlane.

(Str.) Negro, A. (1978) Strumîrs e Zambarlàns. Udine, Societât Filologiiche Furlane.

(Mich.) Michelutti, G. (1978) La Mari di Vigjüt. Udine, Societât Filologiiche Furlane.

(P.) $(1997,1998,1999,2000)$ La Patrie dal Friûl. Gemona.

\section{Bibliografia}

Borghesan, B. (1987) Le forme surcomposées in francese e in altre lingue romanze (tesi di laurea). Udine.

Caput, J.- P. / Caput, J. (1969) Dictionnaire des verbes français. Paris, Larousse.

Castro, F. F. (1999) Las perifrasis verbales en el español actual. Madrid, Editorial Gredos, S.A.

Chevalier, J.- C. / Blanche-Benveniste, C. / Arrivé, M. / Peytard, J. (1964) Grammaire Larousse du français contemporain. Paris, Larousse.

Cornu, M. (1953) Les formes surcomposées en français. Bern.

Damourette, J. / Pichon, É. (1911-1936) Des mots à la pensée - Essai de Grammaire de la Langue Française. Tome V. Paris.

Faggin, G. (1997) Grammatica friulana. Campoformido (Udine), Ribis Editore.

Frau, G. (1984) I dialetti del Friuli. Udine.

Gonzáles, J. G. (1992) Perífrasis verbales. Alcobendas (Madrid), Sociedad General Española de Librería, S.A.

Grevisse, M. (1986) Le bon usage. Paris, Éditions DUCULOT.

Iliescu, M. (1972) Le frioulan à partir des dialectes parlés en Roumanie. Paris, Mouton The Hague.

Jereb, E. (1995) Francoska slovnica po naše. Ljubljana, Cankarjeva zalo ba.

Larousse de la grammaire. I. Dictionnaire des difficultées. (1983) Paris, Larousse.

Larousse. Pluridictionnaire. (1977) Paris, Larousse.

Lexikon der Romanistischen Linguistik. Band/Volume III. (1989) Tübingen, Niemayer.

Marcato, C. (1986) "Forme verbali bicomposte ("surcomposées") nelle parlate del veneto". Guida ai dialetti veneti VIII. Padova, CLEUP editore.

Marchetti, G. (1967) Lineamenti di grammatica friulana. Udine.

Markič, J. (1998) "Los valores aspectuales en el español moderno de América en las obras del escritor colombiano Gabriel Garcia Márquez - Aspektualne vrednosti v sodobni ameriški španščini v delih kolumbijskega pisatelja Gabriela Garcie Márqueza". Verba hispanica, VII. Ljubljana, Filozofska fakulteta.

Melotti, F. / Sordi, I. (1998) Dizionario italiano. Milano, RCS Rizzoli Libri S.p.A.

Miklič, T. (1992) Glagolski časi in izražanje casovnih odnosov v konkretnih besedilih. Ljubljana, Filozofska fakulteta.

Nazzi, G. (1995) Dictionnaire Frioulan (français-frioulan/frioulan-français). Capoformido (Udine), Ribis Editore.

Osservatori regjonâl de lenghe e de culture furlanis (1999) La grafie uficiâl de lenghe furlane. Fiume Veneto (PN), GEAP Print s.r.l.

Pirona, G.A. / Carletti, E. / Corgnali, G.B. (1992) Il Nuovo Pirona. Vocabolario Friulano. Udine, Società Filologica Friulana.

Réquédat, F. (1980) Les constructions verbales avec l'infinitif. Paris, Hachette.

Rizzolatti, P. / Vanelli, L. / Benincà, P. / Vicario, F. / Poletto, C. / Bais, M. (1998) Quaderni della grammatica friulana di riferimento. Anno 1998, num. l. Udine, Editrice Universitaria Udinese S.r.1.

Rohlfs, G. (1969) Grammatica storica della lingua italiana e dei suoi dialetti. Sintassi e formazione delle parole. Torino.

Skubic, M. (1997) Romanske jezikovne prvine na zahodni slovenski jezikovni meji. Ljubljana, Filozofska fakulteta.

Skubic, M. (2002) Romanski jeziki. Ljubljana, Filozofska fakulteta.

Torrego, L.G. (1988) Perifrasis verbales. Madrid, Arco/Libros, S.A.

Vicario, F. (1997) I verbi analitici in friulano. Milano, Franco Angeli.

Weinrich, H. (1989) Grammaire textuelle du français. Paris, Les Éditions Didier.

Zof, F. (2000) Gramatiche pratiche de lenghe furlane. Pasian di Prato, Editrice "Leonardo". 
Povzetek

\section{GLAGOL KOT PRVINA STAVKA V FURLANŠČINI IN FRANCOŠČINI}

Prispevek obravnava glagolske perifraze (GP) in glagolske fraze $z$ drugim glagolom $G F(2 G) v$ sodobnem furlanskem knjižnem jeziku in jih primerja $z$ ustreznimi sintagmami v moderni knjižni francoščini. Izhodišče raziskave je postavka, da predstavlja glagolska perifraza v furlanščini posebno skladenjsko kategorijo, ki se jasno razlikuje od semantičnega sredstva, glagolske fraze $\mathrm{z}$ drugim glagolom. Kontekst določa, ali pri glagolski sintagmi prevladuje skladenjska ali semantična dimenzija, v nekaterih primerih lahko namreč ista sintagma deluje v nekem besedilu kot GP, v drugem pa kot GF(2G). Nadaljnji predmet raziskave je klasifikacija GP. Glede na zgradbo se delijo na GP $z$ nedoločnikom, s preteklim deležnikom in z glagolnikom, po vsebini pa v več sklopov: GP za izraz temporalnosti, modalnosti, faznosti, aspektualnosti, vrste glagolskega dejanja, dosege cilja. Tipična GP je tista, katere konstanta (= glagolski element na levi strani sintagme) je glagol v osebni glagolski obliki, značilnost atipične pa je konstanta v neosebni glagolski obliki. Spremenljivka (= glagolski element na desni strani sintagme) je vedno glagol v neosebni glagolski obliki. Primerjava izvirnika Saint-Exupéryjevega Malega princa s furlanskim prevodom ter francoske in furlanske verzije Evangelija po Luku kaže, da furlanščina često poseže po GP na mestih, kjer se francoščina posluži samo polnopomenskega glagola. Skupno značilnost obravnavanih jezikov predstavlja fenomen dvojno zloženih glagolskih časov, medtem ko pozna furlanščina dve GP za izraz trpnika, francoščina pa le eno. Tudi za izraz obligativnosti razpolaga furlanščina $z$ GP s preteklim deležnikom, ki je francoščini neznana. Francoščina pozna le eno GP z glagolnikom, v furlanščini pa obstajata dve. Za izraz (re)iterativa furlanščina uporablja GP, v nasprotju s francoščino, ki se poslužuje predpone. Furlanske sintagme za izraz zanikanega velelnika so francoščini povsem neznane. Pomembna ugotovitev je tudi, da je furlanski GP za izraz progresivnosti ustrezala francoska sintagma enake zgradbe, ki pa je arhaizem, sodobnemu jeziku komajda še poznan. 